\title{
HARDY SPACES ON METRIC MEASURE SPACES WITH GENERALIZED SUB-GAUSSIAN HEAT KERNEL ESTIMATES
}

\author{
LI CHEN
}

\begin{abstract}
Hardy space theory has been studied on manifolds or metric measure spaces equipped with either Gaussian or sub-Gaussian heat kernel behaviour. However, there are natural examples where one finds a mix of both behaviour (locally Gaussian and at infinity sub-Gaussian) in which case the previous theory doesn't apply. Still we define molecular and square function Hardy spaces using appropriate scaling, and we show that they agree with Lebesgue spaces in some range. Besides, counterexamples are given in this setting that the $H^{p}$ space corresponding to Gaussian estimates may not coincide with $L^{p}$. As a motivation for this theory, we show that the Riesz transform maps our Hardy space $H^{1}$ into $L^{1}$.
\end{abstract}

\section{Contents}

1. Introduction

1.1. The setting

1.2. Definitions

1.3. Main results 5

2. Preliminaries 6

2.1. More about sub-Gaussian off-diagonal and pointwise heat kernel estimates 6

2.2. Tent spaces 8

3. The molecular decomposition 9

3.1. The inclusion $\mathbb{H}_{L, \rho \text {, mol }}^{1}(M) \subseteq H_{L, S_{h}^{\rho}}^{1}(M) \cap H^{2}(M) . \quad 9$

3.2. The inclusion $H_{L, S_{h}^{\rho}}^{1}(M) \cap H^{2}(M) \subseteq \mathbb{H}_{L, \rho \text {, mol }}^{1}(M) . \quad 12$

4. Comparison of Hardy spaces and Lebesgue spaces 14

4.1. $\quad$ Equivalence of $L^{p}(M)$ and $H_{L, S_{h}^{p}}^{p}(M)$ for $p_{0}<p<p_{0}^{\prime} \quad 14$

4.2. Counterexamples to $H_{L, S_{h}}^{p}(M)=L^{p}(M) \quad 21$

5. The $H^{1}-L^{1}$ boundedness of Riesz transforms on fractal manifolds 23

$\begin{array}{ll}\text { References } & 27\end{array}$

\section{INTRODUCTION}

The study of Hardy spaces originated in the 1910's and at the very beginning was confined to Fourier series and complex analysis in one variable. Since 1960's, it has been transferred to real analysis in several variables, or more generally to analysis on metric measure spaces. There are many different equivalent definitions of Hardy spaces, which involve suitable maximal functions,

Date: May 10, 2018.

2010 Mathematics Subject Classification. 42B35(primary); 35J05, 58J35, 42B20 (secondary).

Key words and phrases. Hardy spaces, metric measure spaces, heat kernel estimates, Riesz transforms. 
the atomic decomposition, the molecular decomposition, singular integrals, square functions etc. See, for instance, the classical references [22, 17, 15, 35].

More recently, a lot of work has been devoted to the theory of Hardy spaces associated with operators, see for example, $[5,28,38,4]$ and the references therein.

In [5], Auscher, McIntosh and Russ studied Hardy spaces with respect to the Hodge Laplacian on Riemannian manifolds with the doubling volume property by using the Davies-Gaffney type estimates. They defined Hardy spaces of differential forms of all degrees via molecules and square functions, on which the Riesz transform is $H^{p}$ bounded for $1 \leq p \leq \infty$. Comparing with the Lebesgue spaces, it holds that $H^{p} \subset L^{p}$ for $1 \leq p \leq 2$ and $L^{p} \subset H^{p}$ for $p>2$. Moreover, under the assumption of Gaussian heat kernel upper bound, $H^{p}$ coincides $L^{p}$ for $1<p<\infty$.

In [28], Hofmann, Lu, Mitrea, Mitrea and Yan further developed the theory of $H^{1}$ and $B M O$ spaces adapted to a metric measure space $(M, d, \mu)$ with the volume doubling property endowed with a non-negative self-adjoint operator $L$, which generates an analytic semigroup $\left\{e^{-t L}\right\}_{t>0}$ satisfying the so-called Davies-Gaffney estimate: there exist $C, c>0$ such that for any open sets $U_{1}, U_{2} \subset M$, and for every $f_{i} \in L^{2}(M)$ with supp $f_{i} \subset U_{i}, i=1,2$,

$$
\left|<e^{-t L} f_{1}, f_{2}>\right| \leq C \exp \left(-\frac{\operatorname{dist}^{2}\left(U_{1}, U_{2}\right)}{c t}\right)\left\|f_{1}\right\|_{2}\left\|f_{2}\right\|_{2}, \forall t>0,
$$

where dist $\left(U_{1}, U_{2}\right):=\inf _{x \in U_{1}, y \in U_{2}} d(x, y)$. The authors extended results of [5] by obtaining an atomic decomposition of the $H^{1}$ space.

More generally, instead of (1.1), if $M$ satisfies the Davies-Gaffney estimate of order $m$ with $m \geq 2$ : for all $x, y \in M$ and for all $t>0$,

$$
\left\|\mathbb{1}_{B\left(x, t^{1 / m}\right)} e^{-t L} \mathbb{1}_{B\left(y, t^{1 / m}\right)}\right\|_{2 \rightarrow 2} \leq C \exp \left(-c\left(\frac{d(x, y)}{t}\right)^{\frac{m}{m-1}}\right) .
$$

Kunstmann and Uhl $[38,31]$ defined Hardy spaces via square functions and via molecules adapted to (1.2), where the two $H^{1}$ spaces are also equivalent. Here and in the sequel, $B(x, r)$ denotes the ball of centre $x \in M$ and radius $r>0$ and $V(x, r)=\mu(B(x, r))$. In addition, if the $L^{p_{0}}-L^{p_{0}^{\prime}}$ off-diagonal estimates of order $m$ holds: for all $x, y \in M$ and for all $t>0$,

$$
\left\|\mathbb{1}_{B\left(x, t^{1 / m}\right)} e^{-t L} \mathbb{1}_{B\left(y, t^{1 / m}\right)}\right\|_{p_{0} \rightarrow p_{0}^{\prime}} \leq \frac{C}{V^{\frac{1}{p_{0}}-\frac{1}{p_{0}^{\prime}}\left(x, t^{1 / m}\right)}} \exp \left(-c\left(\frac{d(x, y)}{t}\right)^{\frac{m}{m-1}}\right)
$$

with $p_{0}^{\prime}$ the conjugate of $p_{0}$, then the Hardy space $H^{p}$ defined via square functions coincides with $L^{p}$ for $p \in\left(p_{0}, 2\right)$.

However, there are natural examples where, one finds a mix of both behaviours (1.1) and (1.2), in which case the previous Hardy space theory doesn't apply. For example, on fractal manifolds, the heat kernel behaviour is locally Gaussian and at infinity sub-Gaussian (see Section 2.1 for more details). We aim to develop a proper Hardy space theory for this setting. An important motivation for our Hardy spaces theory is to study the Riesz transform on fractal manifolds, where the weak type $(1,1)$ boundedness has recently been proved in a joint work by the author with Coulhon, Feneuil and Russ [14].

In this paper, we work on doubling metric measure spaces endowed with a non-negative selfadjoint operator which satisfy the doubling volume property and the $L^{2}$ off-diagonal estimate with different local and global decay (see $\left(D G_{\rho}\right)$ below). The specific description will be found below in Section 1.1. We define two classes of Hardy spaces in this setting, via molecules and via conical square functions, see Setion 1.2. Both definitions have the scaling adapted to the off-diagonal decay $\left(D G_{\rho}\right)$.

In Section 3, we identify the two different $H^{1}$ spaces. The molecular $H^{1}$ spaces are always convenient spaces to deal with Riesz transform and other sub-linear operators, while the $H^{p}, p \geq 1$, 
spaces defined via conical square functions possess certain good properties like real and complex interpolation. The identification of both spaces gives us a powerful tool to study the Riesz transform, Littlewood-Paley functions, boundary value problems for elliptic operators etc.

In Section 4, we compare the Hardy spaces defined via conical square functions with the Lebesgue spaces. Assuming further an $L^{p_{0}}-L^{p_{0}^{\prime}}$ off-diagonal estimate for some $1 \leq p_{0}<2$ with different local and global decay for the heat semigroup, we show the equivalence of our $H^{p}$ spaces and the Lebesgue spaces $L^{p}$ for $p_{0}<p<p_{0}^{\prime}$. We also justify that the scaling for the Hardy spaces is the right one, by disproving this equivalence of $H^{p}$ and $L^{p}$ for $p$ close to 2 on some fractal Riemannian manifolds. As far as we know, no previous results are known in this direction.

In Section 5, we shall apply our theory to prove that the Riesz transform is $H^{1}-L^{1}$ bounded on fractal manifolds. The proof is inspired by [14] (see [23]for the original proof in the discrete setting), where the integrated estimate for the gradient of the heat kernel plays a crucial role.

In the following, we will introduce our setting, the definitions and the main results more specifically.

Notation Throughout this paper, we denote $u \simeq v$ if $v \lesssim u$ and $u \lesssim v$, where $u \lesssim v$ means that there exists a constant $C$ (independent of the important parameters) such that $u \leq C v$.

For a ball $B \subset M$ with radius $r>0$ and given $\alpha>0$, we write $\alpha B$ as the ball with the same centre and the radius $\alpha r$. We denote $C_{1}(B)=4 B$, and $C_{j}(B)=2^{j+1} B \backslash 2^{j} B$ for $j \geq 2$.

1.1. The setting. We shall assume that $M$ is a metric measure space satisfying the doubling volume property: for any $x \in M$ and $r>0$,

$$
V(x, 2 r) \lesssim V(x, r)
$$

and the $L^{2}$ Davies-Gaffney estimate with different local and global decay for the analytic semigroup $\left\{e^{-t L}\right\}_{t>0}$ generated by the non-negative self-adjoint operator $L$, that is, $\forall x, y \in M$,

$\left(D G_{\rho}\right) \quad\left\|\mathbb{1}_{B(x, t)} e^{-\rho(t) L_{1}} \mathbb{1}_{B(y, t)}\right\|_{2 \rightarrow 2} \lesssim \begin{cases}\exp \left(-c\left(\frac{d(x, y)}{t}\right)^{\frac{\beta_{1}}{\beta_{1}-1}}\right) & 0<t<1, \\ \exp \left(-c\left(\frac{d(x, y)}{t}\right)^{\frac{\beta_{2}}{\beta_{2}-1}}\right), & t \geq 1,\end{cases}$

where $1<\beta_{1} \leq \beta_{2}$ and

$$
\rho(t)=\left\{\begin{array}{lr}
t^{\beta_{1}}, & 0<t<1, \\
t^{\beta_{2}}, & t \geq 1 .
\end{array}\right.
$$

Recall a simple consequence of $(D)$ : there exists $v>0$ such that

$$
\frac{V(x, r)}{V(x, s)} \lesssim\left(\frac{r}{s}\right)^{v}, \forall x \in M, r \geq s>0 .
$$

It follows that

$$
V(x, r) \lesssim\left(1+\frac{d(x, y)}{r}\right)^{v} V(y, r), \forall x \in M, r \geq s>0 .
$$

Therefore,

$$
\int_{d(x, y)<r} \frac{1}{V(x, r)} d \mu(x) \simeq 1, \forall y \in M, r>0 .
$$


If $M$ is non-compact, we also have a reverse inequality of (1.5) (see for instance [25, p. 412]). That is, there exists $v^{\prime}>0$ such that

$$
\frac{V(x, r)}{V(x, s)} \gtrsim\left(\frac{r}{s}\right)^{v^{\prime}}, \forall x \in M, r \geq s>0 .
$$

Also notice that in (1.4), if necessary we may smoothen $\rho(t)$ as

$$
\rho(t)=\left\{\begin{array}{lr}
t^{\beta_{1}}, & \text { if } 0<t \leq 1 / 2, \\
\text { smooth part, } & \text { if } 1 / 2<t<2, \\
t^{\beta_{2}}, & \text { if } t \geq 2 ;
\end{array}\right.
$$

with $\rho^{\prime}(t) \simeq 1$ for $1 / 2<t<2$, which we still denote by $\rho(t)$. Since $\frac{\rho^{\prime}(t)}{\rho(t)}=\frac{\beta_{1}}{t}$ for $0<t \leq 1 / 2$ and $\frac{\rho^{\prime}(t)}{\rho(t)}=\frac{\beta_{2}}{t}$ for $t \geq 2$, we have in a uniform way

$$
\frac{\rho^{\prime}(t)}{\rho(t)} \simeq \frac{1}{t}
$$

We say that $M$ satisfies an $L^{p_{0}}-L^{p_{0}^{\prime}}$ off-diagonal estimate for some $1<p_{0}<2$ if

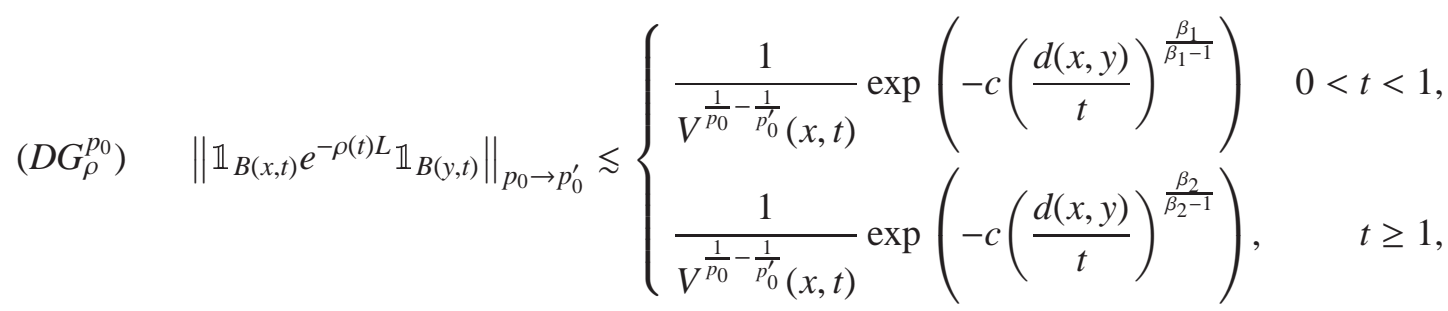

and a generalized pointwise sub-Gaussian heat kernel estimate if for all $x, y \in M$,

$\left(U E_{\rho}\right)$

$$
p_{\rho(t)}(x, y) \lesssim \begin{cases}\frac{1}{V(x, t)} \exp \left(-c\left(\frac{d(x, y)}{t}\right)^{\frac{\beta_{1}}{\beta_{1}-1}}\right) & 0<t<1, \\ \frac{1}{V(x, t)} \exp \left(-c\left(\frac{d(x, y)}{t}\right)^{\frac{\beta_{2}}{\beta_{2}-1}}\right), & t \geq 1,\end{cases}
$$

Examples of fractal manifolds satisfy $\left(U E_{\rho}\right)$ with $\beta_{1}=2$ and $\beta_{2}>2$, see Section 2 below for more information.

1.2. Definitions. Recall that

Definition 1.1. Let $\varepsilon>0$ and an integer $K$ be an integer such that $K>\frac{v}{2 \beta_{1}}$, where $v$ is in (1.5). A function $a \in L^{2}(M)$ is called a $(1,2, \varepsilon)$-molecule associated to $L$ if there exist a function $b \in \mathcal{D}(L)$ and a ball $B$ with radius $r_{B}$ such that

(1) $a=L^{K} b$;

(2) It holds that for every $k=0,1, \cdots, K$ and $i=0,1,2, \cdots$, we have

$$
\left\|\left(\rho\left(r_{B}\right) L\right)^{k} b\right\|_{L^{2}\left(C_{i}(B)\right)} \leq \rho^{K}\left(r_{B}\right) 2^{-i \varepsilon} V\left(2^{i} B\right)^{-1 / 2} .
$$

Definition 1.2. We say that $f=\sum_{n=0}^{\infty} \lambda_{n} a_{n}$ is a molecular $(1,2, \varepsilon)$-representation of $f$ if $\left(\lambda_{n}\right)_{n \in \mathbb{N}} \in$ $l^{1}$, each $a_{n}$ is a molecule as above, and the sum converges in the $L^{2}$ sense. We denote the collection of all the functions with a molecular representation by $\mathbb{H}_{L, \rho \text {, mol }}^{1}$, where the norm of $f \in \mathbb{H}_{L, \rho \text {, mol }}^{1}$ is given by

$$
\|f\|_{\mathbb{H}_{L, \rho, \mathrm{mol}}^{1}(M)}=\inf \left\{\sum_{n=0}^{\infty}\left|\lambda_{n}\right|: f=\sum_{n=0}^{\infty} \lambda_{n} a_{n} \text { is a molecular }(1,2, \varepsilon)-\text { representation }\right\} .
$$


The Hardy space $H_{L, \rho \text {, mol }}^{1}(M)$ is defined as the completion of $\mathbb{H}_{L, \rho, \text { mol }}^{1}(M)$ with respect to this norm.

Consider the following conical square function

$$
S_{h}^{\rho} f(x)=\left(\iint_{\Gamma(x)}\left|\rho(t) L e^{-\rho(t) L} f(y)\right|^{2} \frac{d \mu(y)}{V(x, t)} \frac{d t}{t}\right)^{1 / 2},
$$

where the cone $\Gamma(x)=\{(y, t) \in M \times(0, \infty): d(y, x)<t\}$.

We define first the $L^{2}(M)$ adapted Hardy space $H^{2}(M)$ as the closure of the range of $L$ in $L^{2}(M)$ norm, i.e., $H^{2}(M):=\overline{R(L)}$.

Definition 1.3. The Hardy space $H_{L, S_{h}^{\rho}}^{p}(M), p \geq 1$ is defined as the completion of the set $\{f \in$ $\left.H^{2}(M):\left\|S_{h}^{\rho} f\right\|_{L^{p}}<\infty\right\}$ with respect to the norm $\left\|S_{h}^{\rho} f\right\|_{L^{p}}$. The $H_{L, S_{h}^{\rho}}^{p}(M)$ norm is defined by $\|f\|_{H_{L, S_{h}^{p}}^{p}(M)}:=\left\|S_{h}^{\rho} f\right\|_{L^{p}(M)}$.

For $p=2$, the operator $S_{h}^{\rho}$ is bounded on $L^{2}(M)$. Indeed, for every $f \in L^{2}(M)$,

$$
\begin{aligned}
\left\|S_{h}^{\rho} f\right\|_{L^{2}(M)}^{2} & =\int_{M} \iint_{\Gamma(x)}\left|\rho(t) L e^{-\rho(t) L} f(y)\right|^{2} \frac{d \mu(y)}{V(x, t)} \frac{d t}{t} d \mu(x) \\
& \simeq \iint_{M \times(0, \infty)}\left|\rho(t) L e^{-\rho(t) L} f(y)\right|^{2} d \mu(y) \frac{d t}{t} \\
& \simeq \iint_{M \times(0, \infty)}\left|\rho(t) L e^{-\rho(t) L} f(y)\right|^{2} d \mu(y) \frac{\rho^{\prime}(t) d t}{\rho(t)} \\
& =\int_{0}^{\infty}<(\rho(t) L)^{2} e^{-2 \rho(t) L} f, f>\frac{\rho^{\prime}(t) d t}{\rho(t)} \simeq\|f\|_{L^{2}(M)}^{2} .
\end{aligned}
$$

Note that the second step follows from Fubini theorem and (1.6) in Section 2.3. The third step is obtained by using the fact (1.8): $\rho^{\prime}(t) / \rho(t) \simeq 1 / t$. The last one is a consequence of spectral theory.

Remark 1.4. The above definitions are similar as in [28] (also [5] for 1-forms on Riemannian manifolds) and $[31,38]$. The difference is that we replace $t^{2}$ or $t^{m}$ by $\rho(t)$ in (1.9) and (1.10).

In the case when $\rho(t)=t^{2}$, we denote $S_{h}^{\rho}$ by $S_{h}$, that is,

$$
S_{h} f(x):=\left(\iint_{\Gamma(x)}\left|t^{2} L e^{-t^{2} L} f(y)\right|^{2} \frac{d \mu(y)}{V(x, t)} \frac{d t}{t}\right)^{1 / 2},
$$

and denote $H_{L, S_{h}^{\rho}}^{p}$ by $H_{L, S_{h}}^{p}$.

1.3. Main results. We first obtain the equivalence between $H^{1}$ spaces defined via molecules and via square functions.

Theorem 1.5. Let $M$ be a metric measure space satisfying the doubling volume property $(D)$ and the $L^{2}$ off-diagonal heat kernel estimate $\left(D G_{\rho}\right)$. Then $H_{L, \rho, \mathrm{mol}}^{1}(M)=H_{L, S_{h}^{\rho}}^{1}(M)$, which we denote by $H_{L, \rho}^{1}(M)$. Moreover,

$$
\|f\|_{H_{L, \rho, \text { mol }}^{1}(M)} \simeq\|f\|_{H_{L, S_{h}^{\rho}}^{1}(M)} .
$$

Now compare $H_{L, S_{h}^{\rho}}^{p}(M)$ and $L^{p}$ for $1<p<\infty$.

Recall that on Riemannian manifold satisfying the doubling volume property $(D)$ and the Gaussian upper bound for the heat kernel of the operator, we have $H_{L, S_{h}}^{p}(M)=L^{p}(M), 1<p<\infty$, see for example [5, Theorem 8.5] for Hardy spaces of 0 -forms on Riemannian manifold. However, in general, the equivalence is not known. It is also proved in $[31,38]$ that if the $L^{p_{0}}-L^{p_{0}^{\prime}}$ off-diagonal 
estimates of order $m$ (1.3) holds, then the Hardy space $H_{S_{h}^{m}}^{p}$ (see Remark 1.4) coincides with $L^{p}$ for $p \in\left(p_{0}, 2\right)$.

Our result in this direction is the following:

Theorem 1.6. Let $M$ be a non-compact metric measure space as above. Let $1 \leq p_{0}<2$ and $\rho$ be as above. Suppose that $M$ satisfies $(D)$ and $\left(D G_{\rho}^{p_{0}}\right)$. Then $H_{L, S_{h}^{\rho}}^{p}(M)=L^{p}(M)$ for $p_{0}<p<p_{0}^{\prime}$.

If one assumes the pointwise heat kernel estimate, then Theorems 1.5 and 1.6 yield the following.

Corollary 1.7. Let $M$ be a non-compact metric measure space satisfying the doubling volume property $(D)$ and the pointwise heat kernel estimate $\left(U E_{\rho}\right)$. Then $H_{L, \rho, \operatorname{mol}}^{1}(M)=H_{L, S_{h}^{\rho}}^{1}(M)$, and $H_{L, S_{h}^{\rho}}^{p}(M)=L^{p}(M)$ for $1<p<\infty$.

In the following theorem, we show that for $1<p<2$, the equivalence may not hold between $L^{p}$ and $H^{p}$ defined via conical square function $S_{h}$ with scaling $t^{2}$. The counterexamples we find are certain Riemannian manifolds satisfying $(D)$ and two-sided sub-Gaussian heat kernel estimate: $\left(U E_{\rho}\right)$ and its reverse, with $\beta_{1}=2$ and $\beta_{2}=m>2$. Notice that in this case, $L$ is the non-negative Laplace-Beltrami operator, which we denote by $\Delta$. For simplicity, we denote $\left(U E_{\rho}\right)$ by $\left(U E_{2, m}\right)$ and the two sided estimate by $\left(H K_{2, m}\right)$. Also, we denote by $H_{\Delta, m, m o l}^{1}$ the $H^{1}$ space defined via molecules $H_{L, \rho, m o l}^{1}, H_{\Delta, S_{h}^{m}}^{p}$ the $H^{p}$ space defined via square functions $H_{L, S_{h}^{\rho}}^{p}$.

Theorem 1.8. Let $M$ be a Riemannian manifold with polynomial volume growth

$$
V(x, r) \simeq r^{d}, r \geq 1,
$$

as well as two-sided sub-Gaussian heat kernel estimate $\left(H K_{2, m}\right)$ with $2<m<d / 2$, that is, $\left(U E_{2, m}\right)$ and the matching lower estimate. Then

$$
L^{p}(M) \subset H_{\Delta, S_{h}}^{p}(M)
$$

doesn't hold for $p \in\left(\frac{d}{d-m}, 2\right)$.

As an application of this Hardy space theory, we have

Theorem 1.9. Let $M$ be a manifold satisfying the doubling volume property $(D)$ and the heat kernel estimate $\left(U E_{2, m}\right), m>2$, that is, the upper bound of $\left(H K_{2, m}\right)$. Then the Riesz transform $\nabla \Delta^{-1 / 2}$ is $H_{\Delta, m}^{1}-L^{1}$ bounded.

Remark 1.10. Recall that under the same assumptions, it is proved in [14] that the Riesz transform is of weak type $(1,1)$ and thus $L^{p}$ bounded for $1<p<2$.

\section{Preliminaries}

2.1. More about sub-Gaussian off-diagonal and pointwise heat kernel estimates. Let us first give some examples that satisfy $\left(D G_{\rho}^{p_{0}}\right)$ with $\beta_{1} \neq \beta_{2}$. More examples of this case are metric measure Dirichlet spaces, which we refer to $[8,37,36,27]$ for details.

Example 1. Fractal manifolds.

Fractal manifolds are built from graphs with a self-similar structure at infinity by replacing the edges of the graph with tubes of length 1 and then gluing the tubes together smoothly at the vertices. For instance, see [10] for the construction of Vicsek graphs. For any $D, m \in \mathbb{R}$ such that $D>1$ and $2<m \leq D+1$, there exist complete connected Riemannian manifolds satisfying $V(x, r) \simeq r^{D}$ for $r \geq 1$ and $\left(U E_{\rho}\right)$ with $\beta_{1}=2$ and $\beta_{2}=m>2$ in (1.4) (see [7] and [14]). 
Example 2. Cable systems (Quantum graphs) (see [39], [9, Section 2]).

Given a weighted graph $(G, E, v)$, we define the cable system $G_{C}$ by replacing each edge of $G$ by a copy of $(0,1)$ joined together at the vertices. The measure $\mu$ on $G_{C}$ is given by $d \mu(t)=v_{x y} d t$ for $t$ in the cable connecting $x$ and $y$, and $\mu$ assigns no mass to any vertex. The distance between two points $x$ and $y$ is given as follows: if $x$ and $y$ are on the same cable, the length is just the usual Euclidean distance $|x-y|$. If they are on different cables, then the distance is $\min \left\{\left|x-z_{x}\right|+d\left(z_{x}, z_{y}\right)+\left|z_{y}-y\right|\right\}$ ( $d$ is the usual graph distance), where the minimum is taken over all vertices $z_{x}$ and $z_{y}$ such that $x$ is on a cable with one end at $z_{x}$ and $y$ is on a cable with one end at $z_{y}$. One takes as the core $C$ the functions in $C\left(G_{C}\right)$ which have compact support and are $C^{1}$ on each cable, and sets

$$
\mathcal{E}(f, f):=\int_{G_{C}}\left|f^{\prime}(t)\right|^{2} d \mu(t)
$$

Let $L$ be the associated non-negative self-adjoint operator associated with $\mathcal{E}$ and $\left\{e^{-t L}\right\}_{t>0}$ be the generated semigroup. Then the associated kernel may satisfies $\left(U E_{\rho}\right)$. For example, the cable graph

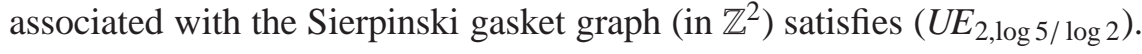

The following are some useful lemmas for the off-diagonal estimates. We first observe that $\left(U E_{\rho}\right)$ $\Rightarrow\left(D G_{\rho}^{p_{0}}\right) \Rightarrow\left(D G_{\rho}\right)$ for $1 \leq p_{0} \leq 2$. Indeed,

Lemma 2.1 ([13]). Let $(M, d, \mu)$ be a metric measure space satisfying the doubling volume property. Let $L$ be a non-negative self-adjoint operator on $L^{2}(M, \mu)$. Assume that $\left(D G_{\rho}^{p_{0}}\right)$ holds. Then for all $p_{0} \leq u \leq v \leq p_{0}^{\prime}$, we have

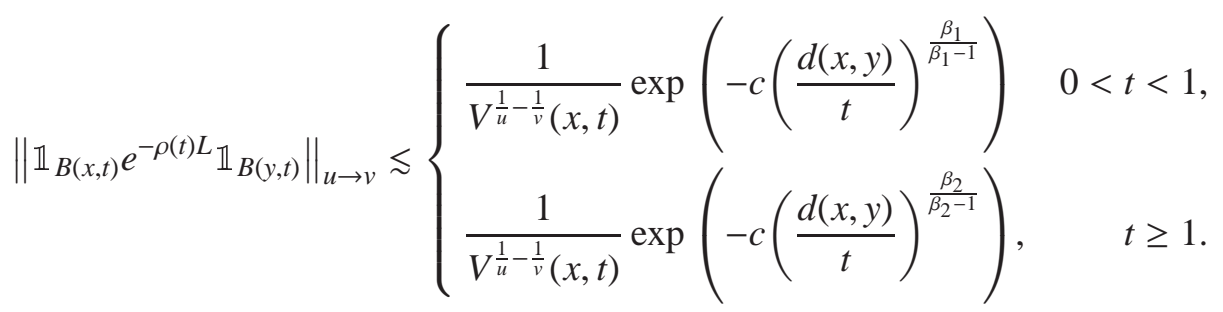

Remark 2.2. The estimate $\left(D G_{\rho}^{p_{0}}\right)$ is equivalent to the $L^{p_{0}}-L^{2}$ off-diagonal estimate

$$
\left\|\mathbb{1}_{B(x, t)} e^{-\rho(t) L} \mathbb{1}_{B(y, t)}\right\|_{p_{0} \rightarrow 2} \lesssim \begin{cases}\frac{1}{V^{\frac{1}{p_{0}}-\frac{1}{2}}(x, t)} \exp \left(-c\left(\frac{d(x, y)}{t}\right)^{\frac{\beta_{1}}{\beta_{1}-1}}\right) & 0<t<1, \\ \frac{1}{V^{\frac{1}{p_{0}}-\frac{1}{2}}(x, t)} \exp \left(-c\left(\frac{d(x, y)}{t}\right)^{\frac{\beta_{2}}{\beta_{2}-1}}\right), \quad t \geq 1 .\end{cases}
$$

We refer to $[13,19]$ for the proof.

In fact, we also have

Lemma 2.3 ([13, 38]). Let $(M, d, \mu)$ satisfy $(D)$. Let L be a non-negative self-adjoint operator on $L^{2}(M, \mu)$. Assume that $\left(D G_{\rho}^{p_{0}}\right)$ holds. Then for all $p_{0} \leq u \leq v \leq p_{0}^{\prime}$ and $k \in \mathbb{N}$, we have

(1) For any ball $B \subset M$ with radius $r>0$, and any $i \geq 2$,

$$
\left\|\mathbb{1}_{B}(t L)^{k} e^{-t L} \mathbb{1}_{C_{i}(B)}\right\|_{u \rightarrow v},\left\|\mathbb{1}_{C_{i}(B)}(t L)^{k} e^{-t L} \mathbb{1}_{B}\right\|_{u \rightarrow v} \lesssim \begin{cases}\frac{2^{i v}}{\mu^{\frac{1}{u}-\frac{1}{v}}(B)} e^{-c\left(\frac{i^{i \beta} \beta_{1}, \beta_{1}}{t}\right)^{1 /\left(\beta_{1}-1\right)}} & 0<t<1, \\ \frac{2^{i v}}{\mu^{\frac{1}{u}-\frac{1}{v}}(B)} e^{-c\left(\frac{i \beta_{2}, \beta_{2}}{t}\right)^{1 /\left(\beta_{2}-1\right)}}, \quad t \geq 1 .\end{cases}
$$


(2) For all $\alpha, \beta \geq 0$ such that $\alpha+\beta=\frac{1}{u}-\frac{1}{v}$,

$$
\left\|V^{\alpha}(\cdot, t)(\rho(t) L)^{k} e^{-\rho(t) L} V^{\beta}(\cdot, t)\right\|_{u \rightarrow v} \leq C .
$$

2.2. Tent spaces. We recall definitions and properties related to tent spaces on metric measure spaces with the doubling volume property, following [15], [33].

Let $M$ be a metric measure space satisfying $(D)$. For any $x \in M$ and for any closed subset $F \subset M$, a saw-tooth region is defined as $\mathcal{R}(F):=\bigcup_{x \in F} \Gamma(x)$. If $O$ is an open subset of $M$, then the "tent" over $O$, denoted by $\widehat{O}$, is defined as

$$
\widehat{O}:=\left[\mathcal{R}\left(O^{c}\right)\right]^{c}=\left\{(x, t) \in M \times(0, \infty): d\left(x, O^{c}\right) \geq t\right\} .
$$

For a measurable function $F$ on $M \times(0, \infty)$, consider

$$
\mathcal{A} F(x)=\left(\iint_{\Gamma(x)}|F(y, t)|^{2} \frac{d \mu(y)}{V(x, t)} \frac{d t}{t}\right)^{1 / 2} .
$$

Given $0<p<\infty$, say that a measurable function $F \in T_{2}^{p}(M \times(0, \infty))$ if

$$
\|F\|_{T_{2}^{p}(M)}:=\|\mathcal{A} F\|_{L^{p}(M)}<\infty .
$$

For simplicity, we denote $T_{2}^{p}(M \times(0, \infty))$ by $T_{2}^{p}(M)$ from now on.

Therefore, for $f \in H_{L, S_{h}}^{p}(M)$ and $0<p<\infty$, write $F(y, t)=\rho(t) L e^{-\rho(t) L} f(y)$, we have

$$
\|f\|_{H_{L, S_{h}^{p}}^{p}(M)}=\|F\|_{T_{2}^{p}(M)} .
$$

Consider another functional

$$
C F(x)=\sup _{x \in B}\left(\iint_{\widehat{B}}|F(y, t)|^{2} \frac{d \mu(y) d t}{t}\right)^{1 / 2},
$$

we say that a measurable function $F \in T_{2}^{\infty}(M)$ if $C F \in L^{\infty}(M)$.

Proposition 2.4. Suppose $1<p<\infty$, let $p^{\prime}$ be the conjugate of $p$. Then the pairing $<F, G>\longrightarrow$ $\int_{M \times(0, \infty)} F(x, t) G(x, t) \frac{d \mu(x) d t}{t}$ realizes $T_{2}^{p^{\prime}}(M)$ as the dual of $T_{2}^{p}(M)$.

Denote by $[,]_{\theta}$ the complex method of interpolation described in [11]. Then we have the following result of interpolation of tent spaces, where the proof can be found in [1].

Proposition 2.5. Suppose $1 \leq p_{0}<p<p_{1} \leq \infty$, with $1 / p=(1-\theta) / p_{0}+\theta / p_{1}$ and $0<\theta<1$. Then

$$
\left[T_{2}^{p_{0}}(M), T_{2}^{p_{1}}(M)\right]_{\theta}=T_{2}^{p}(M) .
$$

Next we review the atomic theory for tent spaces which was originally developed in [15], and extended to the setting of spaces of homogeneous type in [33].

Definition 2.6. A measurable function $A$ on $M \times(0, \infty)$ is said to be a $T_{2}^{1}$-atom if there exists a ball $B \in M$ such that $A$ is supported in $\widehat{B}$ and

$$
\int_{M \times(0, \infty)}|A(x, t)|^{2} d \mu(x) \frac{d t}{t} \leq \mu^{-1}(B) .
$$

Proposition 2.7 ([28],[33]). For every element $F \in T_{2}^{1}(M)$ there exist a sequence of numbers $\left\{\lambda_{j}\right\}_{j=0}^{\infty} \in l^{1}$ and a sequence of $T_{2}^{1}-$ atoms $\left\{A_{j}\right\}_{j=0}^{\infty}$ such that

$$
F=\sum_{j=0}^{\infty} \lambda_{j} A_{j} \text { in } T_{2}^{1}(M) \text { and a.e. in } M \times(0, \infty) \text {. }
$$


Moreover, $\sum_{j=0}^{\infty} \lambda_{j} \approx\|F\|_{T_{2}^{1}(M)}$, where the implicit constants depend only on the homogeneous space properties of $M$.

Finally, if $F \in T_{2}^{1}(M) \cap T_{2}^{2}(M)$, then the decomposition (2.3) also converges in $T_{2}^{2}(M)$.

\section{The MOLECULAR DECOMPOSITION}

In this section, we shall prove Theorem 1.5. That is, under the assumptions of $(D)$ and $\left(D G_{\rho}\right)$, the two $H^{1}$ spaces: $H_{L, \rho, \operatorname{mol}}^{1}(M)$ and $H_{L, S_{h}^{\rho}}^{1}(M)$, are equivalent. We denote

$$
H_{L, \rho}^{1}(M):=H_{L, S_{h}^{\rho}}^{1}(M)=H_{L, \rho, \mathrm{mol}}^{1}(M) .
$$

Since $H_{L, \rho \text {, mol }}^{1}(M)$ and $H_{L, S_{h}^{\rho}}^{1}(M)$ are completions of $\mathbb{H}_{L, \rho \text {, mol }}^{1}(M)$ and $H_{L, S_{h}^{\rho}}^{1}(M) \cap H^{2}(M)$, it is enough to show $\mathbb{H}_{L, \rho, \mathrm{mol}}^{1}(M)=H_{L, S_{h}^{\rho}}^{1}(M) \cap H^{2}(M)$ with equivalent norms. In the following, we will prove the two-sided inclusions seperately. Before proceeding to the proof, we first note the lemma below to prove $H_{L, \rho, \text { mol }}^{1}(M)-L^{1}(M)$ boundedness of an operator, which is an analogue of Lemma 4.3 in [28].

Lemma 3.1. Assume that $T$ is a linear operator, or a nonnegative sublinear operator, satisfying the weak-type $(2,2)$ bound

$$
\mu(\{x \in M:|T f(x)|>\eta\}) \lesssim \eta^{-2}\|f\|_{2}^{2}, \forall \eta>0
$$

and that for every $(1,2, \varepsilon)-$ molecule $a$, we have

$$
\|T a\|_{L^{1}} \leq C,
$$

with constant $C$ independent of $a$. Then $T$ is bounded from $\mathbb{H}_{L, \rho, \operatorname{mol}}^{1}(M)$ to $L^{1}(M)$ with

$$
\|T f\|_{L^{1}} \lesssim\|f\|_{\mathbb{H}_{L, \rho, \mathrm{mol}}^{1}(M)} \text {. }
$$

Consequently, by density, $T$ extends to be a bounded operator from $H_{L, \rho, \text { mol }}^{1}(M)$ to $L^{1}(M)$.

For the proof, we refer to [28], which is also applicable here.

3.1. The inclusion $\mathbb{H}_{L, \rho \text {, mol }}^{1}(M) \subseteq H_{L, S_{h}^{\rho}}^{1}(M) \cap H^{2}(M)$. We have the following theorem:

Theorem 3.2. Let $M$ be a metric measure space satisfying the doubling volume property $(D)$ and the heat kernel estimate $\left(D G_{\rho}\right)$. Then $\mathbb{H}_{L, \rho \text {, mol }}^{1}(M) \subseteq H_{L, S_{h}^{\rho}}^{1}(M) \cap H^{2}(M)$ and

$$
\|f\|_{H_{L, S_{h}^{1}}^{1}(M)} \leq C\|f\|_{\mathbb{H} \mathbb{H}_{L, \rho, \mathrm{mol}}^{1}(M)} \text {. }
$$

Proof. First observe that $\mathbb{H}_{L, \rho \text {, mol }}^{1}(M) \subseteq H^{2}(M)$. Indeed, by Definition 1.1, any $(1,2, \varepsilon)$-molecule belongs to $R(L)$. Thus any finite linear combination of molecules belongs to $R(L)$. Since $f \in$ $\mathbb{H}_{L, \rho, \text { mol }}^{1}(M)$ is the $L^{2}(M)$ limit of finite linear combination of molecules, we get $f \in \overline{R(L)}=H^{2}(M)$.

It remains to show $\mathbb{H}_{L, \rho \text {, mol }}^{1}(M) \subseteq H_{L, S_{h}^{\rho}}^{1}(M)$, that is, $S_{h}^{\rho}$ is bounded from $\mathbb{H}_{L, \rho \text {, mol }}^{1}(M)$ to $L^{1}(M)$. Note that $S_{h}^{\rho}$ is $L^{2}$ bounded by spectral theory (see (1.11)), it follows from Lemma 3.1 that it suffices to prove that, for any $(1,2, \varepsilon)$-molecule $a$, there exists a constant $C$ such that $\left\|S_{h}^{\rho} a\right\|_{L^{1}(M)} \leq C$. In other words, one needs to prove $\|A\|_{T_{2}^{1}(M)} \leq C$, where

$$
A(y, t)=\rho(t) L e^{-\rho(t) L} a(y) .
$$

Assume that $a$ is a $(1,2, \varepsilon)$-molecule related to a function $b$ and a ball $B$ with radius $r$, that is, $a=L^{K} b$ and for every $k=0,1, \cdots, K$ and $i=0,1,2, \cdots$, it holds that

$$
\left\|(\rho(r) L)^{k} b\right\|_{L^{2}\left(C_{i}(B)\right)} \leq \rho(r) 2^{-i \varepsilon} \mu\left(2^{i} B\right)^{-1 / 2} .
$$


Similarly as in [5], we divide $A$ into four parts:

$$
\begin{aligned}
A & =\mathbb{1}_{2 B \times(0,2 r)} A+\sum_{i \geq 1} \mathbb{1}_{C_{i}(B) \times(0, r)} A+\sum_{i \geq 1} \mathbb{1}_{C_{i}(B) \times\left(r, 2^{i+1} r\right)} A+\sum_{i \geq 1} \mathbb{1}_{2^{i} B \times\left(2^{i}, 2^{i+1} r\right)} A \\
& =: A_{0}+A_{1}+A_{2}+A_{3} .
\end{aligned}
$$

Here $\mathbb{1}$ denotes the characteristic function and $C_{i}(B)=2^{i+1} B \backslash 2^{i}(B), i \geq 1$. It suffices to show that for every $j=0,1,2,3$, we have $\left\|A_{j}\right\|_{T_{2}^{1}} \leq C$.

Firstly consider $A_{0}$. Observe that

$$
\mathcal{A}\left(A_{0}\right)(x)=\left(\iint_{\Gamma(x)}\left|\mathbb{1}_{2 B \times(0,2 r)}(y, t) A(y, t)\right|^{2} \frac{d \mu(y)}{V(x, t)} \frac{d t}{t}\right)^{1 / 2}
$$

is supported on $4 B$. Indeed, denote by $x_{B}$ be the center of $B$, then $d\left(x, x_{B}\right) \leq d(x, y)+d\left(y, x_{B}\right) \leq 4 r$. Also, it holds that

$$
\begin{aligned}
\left\|A_{0}\right\|_{T_{2}^{2}(M)}^{2} & =\left\|\mathcal{A}\left(A_{0}\right)\right\|_{2}^{2} \leq \int_{M} \iint_{\Gamma(x)}\left|\rho(t) L e^{-\rho(t) L} a(y)\right|^{2} \frac{d \mu(y)}{V(x, t)} \frac{d t}{t} d \mu(x) \\
& \lesssim\|a\|_{L^{2}(M)}^{2} \lesssim \mu^{-1}(B) .
\end{aligned}
$$

Here the second and the third inequalities follow from (1.11) and the definition of molecules respectively. Now applying the Cauchy-Schwarz inequality, then

$$
\left\|A_{0}\right\|_{T_{2}^{1}(M)} \leq\|A\|_{T_{2}^{2}(M)} \mu(4 B)^{1 / 2} \leq C .
$$

Secondly for $A_{1}$. For each $i \geq 1$, we have supp $\mathcal{A}\left(\mathbb{1}_{C_{i}(B) \times(0, r)} A\right) \subset 2^{i+2} B$. In fact, $d\left(x, x_{B}\right) \leq$ $d(x, y)+d\left(y, x_{B}\right) \leq t+2^{i+1} r<2^{i+2} r$. Then

$$
\begin{aligned}
\left\|\mathbb{1}_{C_{i}(B) \times(0, r)} A\right\|_{T_{2}^{2}} & =\left\|\mathcal{A}\left(\mathbb{1}_{C_{i}(B) \times(0, r)} A\right)\right\|_{2} \\
& \leq\left(\int_{2^{i+2} B} \iint_{\Gamma(x)}\left|\mathbb{1}_{C_{i}(B) \times\left(0, r_{B}\right)}(y, t) \rho(t) L e^{-\rho(t) L} a(y)\right|^{2} \frac{d \mu(y)}{V(x, t)} \frac{d t}{t} d \mu(x)\right)^{1 / 2} \\
& \leq\left(\int_{0}^{r} \int_{C_{i}(B)}\left|\rho(t) L e^{-\rho(t) L} a(y)\right|^{2} d \mu(y) \frac{d t}{t}\right)^{1 / 2} \\
& \leq \sum_{l=0}^{\infty}\left(\int_{0}^{r} \int_{C_{i}(B)}\left|\rho(t) L e^{-\rho(t) L_{1}} \mathbb{1}_{C_{l}(B)} a(y)\right|^{2} d \mu(y) \frac{d t}{t}\right)^{1 / 2} \\
& =: \sum_{l=0}^{\infty} I_{l} .
\end{aligned}
$$

We estimate $I_{l}$ with $|i-l|>3$ and $|i-l| \leq 3$ respectively. Firstly assume that $|i-l| \leq 3$. Using (1.11) again, we have

$$
I_{l}^{2} \leq \int_{0}^{\infty} \int_{M}\left|\rho(t) L e^{-\rho(t) L} \mathbb{1}_{C_{l}(B)} a(y)\right|^{2} d \mu(y) \frac{d t}{t} \lesssim\|a\|_{L^{2}\left(C_{l}(B)\right)}^{2} \lesssim 2^{-2 i \varepsilon} \mu^{-1}\left(2^{i} B\right) .
$$

Assume now $|i-l|>3$. Note that $\operatorname{dist}\left(C_{l}(B), C_{i}(B)\right) \geq c 2^{\max \{l, i\}} r_{B} \geq c 2^{i} r_{B}$. Then it follows from Lemma 2.3 that

$$
\begin{aligned}
I_{l}^{2} & \leq \int_{0}^{r} \exp \left(-c\left(\frac{\rho\left(2^{i} r\right)}{\rho(t)}\right)^{\frac{\beta_{2}}{\beta_{2}-1}}\right)\|a\|_{L^{2}\left(C_{l}(B)\right)}^{2} d \mu(y) \frac{d t}{t} \\
& \lesssim 2^{-2 l \varepsilon} \mu^{-1}\left(2^{l} B\right) \int_{0}^{r}\left(\frac{\rho(t)}{\rho\left(2^{i} r\right)}\right)^{c} \frac{d t}{t} \lesssim 2^{-c i} 2^{-2 l \varepsilon} \mu^{-1}\left(2^{i} B\right) .
\end{aligned}
$$

The last inequality follows from (1.5). 
It follows from above that

$$
\left\|\mathbb{1}_{C_{i}(B) \times(0, r)} A\right\|_{T_{2}^{2}} \lesssim \sum_{l:|l-i| \leq 3} 2^{-i \varepsilon} \mu^{-1 / 2}\left(2^{i} B\right)+\sum_{l:|l-i|>3} 2^{-i c} 2^{-l \varepsilon} \mu^{-1 / 2}\left(2^{i} B\right) \lesssim 2^{-i c} \mu^{-1 / 2}\left(2^{i} B\right),
$$

where $c$ depends on $\varepsilon, M$. Therefore

$$
\left\|A_{1}\right\|_{T_{2}^{1}} \leq \sum_{i \geq 1}\left\|\mathbb{1}_{C_{i}(B) \times(0, r)} A\right\|_{T_{2}^{2}} \mu^{1 / 2}\left(2^{i+2} B\right) \lesssim \sum_{i \geq 1} 2^{-i c} \leq C .
$$

We estimate $A_{2}$ in a similar way as before except that we replace $a$ by $L^{K} b$. Note that for each $i \leq 1$, we have supp $\mathcal{A}\left(\mathbb{1}_{C_{i}(B) \times\left(r, 2^{i+1} r\right)} A\right) \subset 2^{i+2} B$. Indeed,

$$
d\left(x, x_{B}\right) \leq d(x, y)+d\left(y, x_{B}\right) \leq t+2^{i+1} r \leq 2^{i+2} r .
$$

Then

$$
\begin{aligned}
\left\|\mathbb{1}_{C_{i}(B) \times\left(r, 2^{i+1} r\right)} A\right\|_{T_{2}^{2}} & =\left\|\mathcal{A}\left(\mathbb{1}_{C_{i}(B) \times\left(r, 2^{i+1} r\right)} A\right)\right\|_{2} \\
& \leq\left(\int_{2^{i+2} B} \iint_{\Gamma(x)}\left|\mathbb{1}_{C_{i}(B) \times\left(r, 2^{i} r\right)}(y, t) A(y, t)\right|^{2} \frac{d \mu(y) d t}{V(x, t) t} d \mu(x)\right)^{1 / 2} \\
& \leq\left(\int_{r}^{2^{i+1} r} \int_{C_{i}(B)}\left|(\rho(t) L)^{K+1} e^{-\rho(t) L} b(y)\right|^{2} d \mu(y) \frac{d t}{t \rho^{2 K}(t)}\right)^{1 / 2} \\
& \leq\left(\sum_{l=0}^{\infty} \int_{r}^{2^{i+1} r} \int_{C_{i}(B)}\left|(\rho(t) L)^{K+1} e^{-\rho(t) L_{1}} \mathbb{1}_{C_{l}(B)} b(y)\right|^{2} d \mu(y) \frac{d t}{t \rho^{2 K}(t)}\right)^{1 / 2} \\
& =: \sum_{l=0}^{\infty} J_{l}
\end{aligned}
$$

When $|i-l| \leq 3$, by spectral theorem we get $J_{l}^{2} \leq C 2^{-2 i \varepsilon} V^{-1}\left(2^{i} B\right)$. And when $|i-l|>3$, it holds $\operatorname{dist}\left(C_{l}(B), C_{i}(B)\right) \geq c 2^{\max \{l, i\}} r \geq c 2^{i} r$. Then we estimate $J_{l}$ in the same way as for (3.3),

$$
\begin{aligned}
J_{l}^{2} & \leq \int_{r}^{2^{i+1} r} \exp \left(-c\left(\frac{\rho\left(2^{i} r\right)}{\rho(t)}\right)^{\frac{\beta_{2}}{\beta_{2}-1}}\right)\|b\|_{L^{2}\left(C_{l}(B)\right)}^{2} d \mu(y) \frac{d t}{t \rho^{2 K}(t)} \\
& \leq \rho^{2 K}(r) 2^{-2 l \varepsilon} \mu^{-1}\left(2^{l+1} B\right) \int_{r}^{2^{i+1} r}\left(\frac{\rho(t)}{\rho\left(2^{i} r\right)}\right)^{c} \frac{d t}{t \rho^{2 K}(t)} \\
& \lesssim 2^{-i c} 2^{-l(2 \varepsilon+v)} \mu^{-1}\left(2^{i} B\right) .
\end{aligned}
$$

Here $c$ in the second and the third lines are different. We can carefully choose $c$ in the second line to make sure that $c$ in the third line is positive.

Hence

$$
\left\|\mathbb{1}_{C_{i}(B) \times\left(r, 2^{i} r\right)} A\right\|_{T_{2}^{2}}^{2} \lesssim 2^{-i c} \mu^{-1}\left(2^{i} B\right),
$$

and

$$
\left\|A_{2}\right\|_{T_{2}^{1}} \leq \sum_{i \geq 1}\left\|\mathbb{1}_{C_{i}(B) \times\left(r, 2^{i} r\right)} A\right\|_{T_{2}^{2}} \mu^{1 / 2}\left(2^{i+2} B\right) \lesssim \sum_{i \geq 1} 2^{-i c / 2} \leq C .
$$

It remains to estimate the last term $A_{3}$. For each $i \geq 1$, we still have

$$
\operatorname{supp} \mathcal{A}\left(\mathbb{1}_{2^{i} B \times\left(2^{i} r, 2^{i+1} r\right)} A\right) \subset 2^{i+2} B \text {. }
$$

Then we obtain as before that

$$
\left\|\mathbb{1}_{2^{i} B \times\left(2^{i} r 2^{i+1} r\right)} A\right\|_{T_{2}^{2}}=\left\|\mathcal{A}\left(\mathbb{1}_{2^{i} B \times\left(2^{i} r, 2^{i+1} r\right)} A\right)\right\|_{2}
$$




$$
\begin{aligned}
& \leq\left(\int_{2^{i+2} B} \iint_{\Gamma(x)}\left|\mathbb{1}_{2^{i} B \times\left(2^{i} r_{B}, 2^{i+1} r\right)}(y, t) A(y, t)\right|^{2} \frac{d \mu(y) d t}{V(x, t) t} d \mu(x)\right)^{1 / 2} \\
& \leq\left(\int_{2^{i} r}^{2^{i+1} r} \int_{2^{i} B}\left|(\rho(t) L)^{K+1} e^{-\rho(t) L} b(y)\right|^{2} \frac{d \mu(y) d t}{t \rho^{2 K}(t)}\right)^{1 / 2} \\
& \leq \sum_{l=0}^{\infty}\left(\int_{2^{i} r}^{2^{i+1} r} \int_{2^{i} B}\left|(\rho(t) L)^{2} e^{-\rho(t) L} \mathbb{1}_{C_{l}(B)} b(y)\right|^{2} \frac{d \mu(y) d t}{t \rho^{2 K}(t)}\right)^{1 / 2} \\
& =: \sum_{l=0}^{\infty} K_{l} .
\end{aligned}
$$

In fact, due to the doubling volume property, (1.11) as well as the definition of molecules, we get

$$
\begin{aligned}
K_{l}^{2} & \leq \int_{2^{i} r}^{2^{i+1} r}\left\|\mathbb{1}_{C_{l}(B)} b\right\|_{L^{2}}^{2} \frac{d t}{t \rho^{2 K}(t)} \lesssim \rho^{2 K}(r) 2^{-2 l \varepsilon} \mu^{-1}\left(2^{l} B\right) \int_{2^{i} r}^{2^{i+1} r} \frac{d t}{t \rho^{2 K}(t)} \\
& \lesssim 2^{-2 l \varepsilon} 2^{-i c} \mu^{-1}\left(2^{i} B\right) .
\end{aligned}
$$

Hence

$$
\left\|A_{3}\right\|_{T_{2}^{1}} \leq \sum_{i \geq 1}\left\|\mathbb{1}_{2^{i} B \times\left(2^{i} r, 2^{i+1} r\right)} A\right\|_{T_{2}^{2}} \mu^{1 / 2}\left(2^{i+2} B\right) \lesssim \sum_{i \geq 1} 2^{-2 i} \leq C .
$$

This finishes the proof.

3.2. The inclusion $H_{L, S_{h}^{\rho}}^{1}(M) \cap H^{2}(M) \subseteq \mathbb{H}_{L, \rho \text {, mol }}^{1}(M)$. We closely follow the proof of Theorem 4.13 in [28] and get

Theorem 3.3. Let $M$ be a metric measure space satisfying $(D)$ and $\left(D G_{\rho}\right)$. If $f \in H_{L, S_{h}^{\rho}}^{1}(M) \cap$ $H^{2}(M)$, then there exist a sequence of numbers $\left\{\lambda_{j}\right\}_{j=0}^{\infty} \subset l^{1}$ and a sequence of $(1,2, \varepsilon)$-molecules $\left\{a_{j}\right\}_{j=0}^{\infty}$ such that $f$ can be represented in the form $f=\sum_{j=0}^{\infty} \lambda_{j} a_{j}$, with the sum converging in $L^{2}(M)$, and

$$
\|f\|_{\mathbb{H}_{L, \rho, \mathrm{mol}}^{1}(M)} \leq C \sum_{j=0}^{\infty} \lambda_{j} \leq C\|f\|_{H_{L, S_{h}^{\rho}}^{1}(M)},
$$

where $C$ is independent of $f$. In particular, $H_{L, S_{h}^{\rho}}^{1}(M) \cap H^{2}(M) \subseteq \mathbb{H}_{L, \rho \text {, mol }}^{1}(M)$.

Proof. For $f \in H_{L, S_{h}^{\rho}}^{1}(M) \cap H^{2}(M)$, denote $F(x, t)=\rho(t) L e^{-\rho(t) L} f(x)$. Then by the definition of $H_{L, S_{h}^{\rho}}^{1}(M)$, we have $F \in T_{2}^{1}(M) \cap T_{2}^{2}(M)$.

From Theorem 2.7, we decompose $F$ as $F=\sum_{j=0}^{\infty} \lambda_{j} A_{j}$, where $\left\{\lambda_{j}\right\}_{j=0}^{\infty} \in l^{1},\left\{A_{j}\right\}_{j=0}^{\infty}$ is a sequence of $T_{2}^{1}$-atoms supported in a sequence of sets $\left\{\widehat{B}_{j}\right\}_{j=0}^{\infty}$, and the sum converges in both $T_{2}^{1}(M)$ and $T_{2}^{2}(M)$. Also

$$
\sum_{j=0}^{\infty} \lambda_{j} \lesssim\|F\|_{T_{2}^{1}(X)}=\|f\|_{H_{L, S_{h}^{\rho}}^{1}(M)} .
$$

For $f \in H^{2}(M)$, by functional calculus, we have the following "Calderón reproducing formula"

$$
f=C \int_{0}^{\infty}(\rho(t) L)^{K+1} e^{-2 \rho(t) L} f \frac{\rho^{\prime}(t) d t}{\rho(t)}=C \int_{0}^{\infty}(\rho(t) L)^{K} e^{-\rho(t) L} F(\cdot, t) \frac{\rho^{\prime}(t) d t}{\rho(t)}=: C \pi_{h, L}(F) .
$$

Denote $a_{j}=C \pi_{h, L}\left(A_{j}\right)$, then $f=\sum_{j=0}^{\infty} \lambda_{j} a_{j}$. Since for $F \in T_{2}^{2}(M)$, we have $\left\|\pi_{h, L}(F)\right\|_{L^{2}(M)} \leq$ $C\|F\|_{T_{2}^{2}(M)}$. Thus we learn from Lemma 4.12 in [28] that the sum also converges in $L^{2}(M)$. 
We claim that $a_{j}, j=0,1, \ldots$, are $(1,2, \varepsilon)$-molecules up to multiplication to some uniform constant.

Indeed, note that $a_{j}=L^{K} b_{j}$, where

$$
b_{j}=C \int_{0}^{\infty} \rho^{K}(t) e^{-\rho(t) L} A_{j}(\cdot, t) \frac{\rho^{\prime}(t) d t}{\rho(t)} .
$$

Now we estimate the norm $\left\|\left(\rho\left(r_{B_{j}}\right) L\right)^{k} b_{j}\right\|_{L^{2}\left(C_{i}(B)\right)}$, where $r_{B_{j}}$ is the radius of $B_{j}$. For simplicity we ignore the index $j$. Consider any function $g \in L^{2}\left(C_{i}(B)\right)$ with $\|g\|_{L^{2}\left(C_{i}(B)\right)}=1$, then for $k=$ $0,1, \cdots, K$,

$$
\begin{aligned}
& \left|\int_{M}\left(\rho\left(r_{B}\right) L\right)^{k} b(x) g(x) d \mu(x)\right| \\
& \lesssim\left|\int_{M}\left(\int_{0}^{\infty}\left(\rho\left(r_{B}\right) L\right)^{k} \rho^{K}(t) e^{-\rho(t) L}\left(A_{j}(\cdot, t)\right)(x) \frac{\rho^{\prime}(t) d t}{\rho(t)}\right) g(x) d \mu(x)\right| \\
& =\left|\int_{\widehat{B}}\left(\frac{\rho\left(r_{B}\right)}{\rho(t)}\right)^{k} \rho^{K}(t) A_{j}(x, t)(\rho(t) L)^{k} e^{-\rho(t) L} g(x) d \mu(x) \frac{\rho^{\prime}(t) d t}{\rho(t)}\right| \\
& \lesssim\left(\int_{\widehat{B}}\left|A_{j}(x, t)\right|^{2} d \mu(x) \frac{d t}{t}\right)^{1 / 2}\left(\int_{\widehat{B}}\left|\left(\frac{\rho\left(r_{B}\right)}{\rho(t)}\right)^{k} \rho^{K}(t)(\rho(t) L)^{k} e^{-\rho(t) L} g(x)\right|^{2} d \mu(x) \frac{d t}{t}\right)^{1 / 2} .
\end{aligned}
$$

In the last inequality, we apply Hölder inequality as well as (1.8).

We continue to estimate by using the definition of $T_{2}^{1}$-atoms and the off-diagonal estimates of heat kernel.

For $i=0,1$, the above quantity is dominated by

$$
\mu^{-1 / 2}(B) \rho\left(r_{B}\right)\left(\int_{\widehat{B}}\left|(\rho(t) L)^{k} e^{-\rho(t) L} g(x)\right|^{2} d \mu(x) \frac{d t}{t}\right)^{1 / 2} \lesssim \mu^{-1 / 2}(B) \rho\left(r_{B}\right)
$$

Next for $i \geq 2$, the above estimate is controlled

$$
\begin{aligned}
& \mu^{-1 / 2}(B)\left(\int_{0}^{r_{B}}\left(\frac{\rho\left(r_{B}\right)}{\rho(t)}\right)^{2 k} \rho^{2 K}(t)\left\|(\rho(t) L)^{k} e^{-\rho(t) L} g\right\|_{L^{2}(B)}^{2} \frac{d t}{t}\right)^{1 / 2} \\
& \lesssim \mu^{-1 / 2}(B)\left(\int_{0}^{r_{B}}\left(\frac{\rho\left(r_{B}\right)}{\rho(t)}\right)^{2 k} \rho^{2 K}(t) \exp \left(-c\left(\frac{2^{i} r_{B}}{t}\right)^{\tau}\right) \frac{d t}{t}\right)^{1 / 2} \\
& \lesssim \mu^{-1 / 2}(B)\left(\int_{0}^{r_{B}}\left(\frac{\rho\left(r_{B}\right)}{\rho(t)}\right)^{2 k} \rho^{2 K}(t)\left(\frac{t}{2^{i} r_{B}}\right)^{\varepsilon+v} \frac{d t}{t}\right)^{1 / 2} \\
& \lesssim \mu^{-1 / 2}\left(2^{i} B\right) \rho^{K}\left(r_{B}\right) 2^{-i \varepsilon} .
\end{aligned}
$$

In the first inequality, we use Lemma 2.3. Since $k=0,1, \cdots, K$, the last inequality always holds for any $\varepsilon>0$.

Therefore,

$$
\begin{aligned}
\left\|\left(\rho\left(r_{B}\right) L\right)^{k} b\right\|_{L^{2}\left(C_{i}(B)\right)} & =\sup _{\|g\|_{L^{2}\left(C_{i}(B)\right)}}\left|\int_{M}\left(\rho\left(r_{B}\right) L\right)^{k} b(x) g(x) d \mu(x)\right| \\
& \lesssim \mu^{-1 / 2}\left(2^{i} B\right) \rho^{K}\left(r_{B}\right) 2^{-i \varepsilon}
\end{aligned}
$$




\section{Comparison of Hardy sPaces and Lebesgue spaces}

In this section, we will study the relations between $L^{p}(M), H_{L, S_{h}^{\rho}}^{p}(M)$ and $H_{L, S_{h}}^{p}(M)$ under the assumptions of $(D)$ and $\left(D G_{\rho}^{p_{0}}\right)$. We first show that $L^{p}(M)$ and $H_{L, S_{h}^{p}}^{p}(M)$ are equivalent. Next we give some examples such that $L^{p}(M)$ and $H_{L, S_{h}}^{p}(M)$ are not equivalent. More precisely, the inclusion $L^{p} \subset H_{L, S_{h}}^{p}$ may be false for $1<p<2$.

4.1. Equivalence of $L^{p}(M)$ and $H_{L, S^{\rho}}^{p}(M)$ for $p_{0}<p<p_{0}^{\prime}$. We will prove Theorem 1.6. That is, if $M$ satisfies $(D)$ and $\left(D G_{\rho}^{p_{0}}\right)$, then $H_{L, S_{h}^{\rho}}^{p}(M)=L^{p}(M)$ for $p_{0}<p<p_{0}^{\prime}$.

Our main tool is the Calderón-Zygmund decomposition (see for example [16, Corollaire 2.3]).

Theorem 4.1. Let $(M, d, \mu)$ be a metric measured space satisfying the doubling volume property. Let $1 \leq q \leq \infty$ and $f \in L^{q}$. Let $\lambda>0$. Then there exists a decomposition of $f, f=g+b=g+\sum_{i} b_{i}$ so that

(1) $|g(x)| \leq C \lambda$ for almost all $x \in M$;

(2) There exists a sequence of balls $B_{i}=B\left(x_{i}, r_{i}\right)$ so that each $b_{i}$ is supported in $B_{i}$,

$$
\int\left|b_{i}(x)\right|^{q} d \mu(x) \leq C \lambda^{q} \mu\left(B_{i}\right)
$$

(3) $\sum_{i} \mu\left(B_{i}\right) \leq \frac{C}{\lambda^{q}} \int|f(x)|^{q} d \mu(x)$;

(4) $\|b\|_{q} \leq C\|f\|_{q}$ and $\|g\|_{q} \leq C\|f\|_{q}$;

(5) There exists $k \in \mathbb{N}^{*}$ such that each $x \in M$ is contained in at most $k$ balls $B_{i}$.

Proof of Theorem 1.6. Due to the self-adjointness of $L$ in $L^{2}(M)$, we get $L^{2}(M)=\overline{R(L)} \bigoplus N(L)$, where the sum is orthogonal. Under the assumptions $(D)$ and $\left(D G_{\rho}^{p_{0}}\right)$, we have $N(L)=0$ and thus $H^{2}(M)=L^{2}(M)$. Indeed, for any $f \in N(L)$, it holds

$$
e^{-\rho(t) L} f-f=\int_{0}^{\rho(t)} \frac{\partial}{\partial s} e^{-s L} f d s=-\int_{0}^{\rho(t)} L e^{-s L} f d s=0,
$$

As a consequence of Lemma 2.1, we have that for all $x \in M$ and $t \geq 0$,

$$
\left(\int_{B(x, t)}|f|^{p_{0}^{\prime}}\right)^{1 / p_{0}^{\prime}}=\left\|e^{-\rho(t) L} f\right\|_{L^{p_{0}^{\prime}(B(x, t))}} \lesssim V(x, t)^{\frac{1}{p_{0}^{\prime}}-\frac{1}{2}}\|f\|_{L^{2}(B(x, t))} .
$$

Now letting $t \rightarrow \infty$, we obtain that $f=0$.

It suffices to prove that for any $f \in R(L) \cap L^{p}(M)$ with $p_{0}<p<p_{0}^{\prime}$,

$$
\left\|S_{h}^{\rho} f\right\|_{L^{p}} \lesssim\|f\|_{L^{p}} .
$$

With this fact at hand, we can obtain by duality that $\|f\|_{L^{p}} \leq C\left\|S_{h}^{\rho} f\right\|_{L^{p}}$ for $p_{0}<p<p_{0}^{\prime}$.

Indeed, for $f \in R(L)$, write the identity

$$
f=C \int_{0}^{\infty}(\rho(t) L)^{2} e^{-2 \rho(t) L} f \frac{\rho^{\prime}(t) d t}{\rho(t)},
$$

where the integral $C \int_{\varepsilon}^{1 / \varepsilon}(\rho(t) L)^{2} e^{-2 \rho(t) L} f \frac{\rho^{\prime}(t) d t}{\rho(t)}$ converges to $f$ in $L^{2}(M)$ as $\varepsilon \rightarrow 0$.

Then for $f \in R(L) \cap L^{p}(M)$, we have

$$
\begin{aligned}
\|f\|_{L^{p}} & =\sup _{\|g\|_{L^{p^{\prime}} \leq 1}}|<f, g>| \simeq \sup _{\|g\|_{L^{p^{\prime}}} \leq 1}\left|\iint_{M \times(0, \infty)} F(y, t) G(y, t) d \mu(y) \frac{\rho^{\prime}(t) d t}{\rho(t)}\right| \\
& \simeq \sup _{\|g\|_{L^{p^{\prime}} \leq 1}}\left|\int_{M} \iint_{\Gamma(x)} F(y, t) G(y, t) \frac{d \mu(y)}{V(x, t)} \frac{\rho^{\prime}(t) d t}{\rho(t)} d \mu(x)\right|
\end{aligned}
$$




$$
\begin{aligned}
& \lesssim \sup _{\|g\|_{L^{p^{\prime}} \leq 1}}\|F\|_{T_{2}^{p}}\|G\|_{T_{2}^{p^{\prime}}} \simeq \sup _{\|g\|_{L^{p^{\prime}}} \leq 1}\left\|S_{h} f\right\|_{L^{p}}\left\|S_{h} g\right\|_{L^{p^{\prime}}} \\
& \lesssim \sup _{\|g\|_{L^{p^{\prime}}} \leq 1}\left\|S_{h} f\right\|_{L^{p}}\|g\|_{L^{p^{\prime}}}=\left\|S_{h} f\right\|_{L^{p}} .
\end{aligned}
$$

Here $F(y, t)=\rho(t) L e^{-\rho(t) L} f(y)$ and $G(y, t)=\rho(t) L e^{-\rho(t) L} g(y)$. The second line's equivalence is due to the doubling volume propsimeqerty.

By an approximation process, the above argument holds for $f \in L^{p}(M)$.

For $p>2$, the $L^{p}$ norm of the conical square function is controlled by its vertical analogue (for a reference, see [3], where the proof can be adapted to the homogenous setting), which is always $L^{p}$ bounded for $p_{0}<p<p_{0}^{\prime}$ by adapting the proofs in [12] and [20] (if $\left\{e^{-t L}\right\}_{t>0}$ is a symmetric Markov semigroup, then it is $L^{p}$ bounded for $1<p<\infty$, according to [34]). Hence (4.1) holds.

It remains to show (4.1) for $p_{0}<p<2$.

In the following, we will prove the weak $\left(p_{0}, p_{0}\right)$ boundedness of $S_{h}^{\rho}$ by using the CalderónZygmund decomposition. Since $S_{h}^{\rho}$ is also $L^{2}$ bounded as shown in (1.11), then by interpolation, (4.1) holds for every $p_{0}<p<2$. The proof is similar to [2, Proposition 6.8] and [3, Theorem 3.1], which originally comes from [21].

We take the Calderón-Zygmund decomposition of $f$ at height $\lambda$, that is, $f=g+\sum b_{i}$ with supp $b_{i} \subset B_{i}$. Since $S_{h}^{\rho}$ is a sublinear operator, write

$$
\begin{aligned}
S_{h}^{\rho}\left(\sum_{i} b_{i}\right) & =S_{h}^{\rho}\left(\sum_{i}\left(I-\left(I-e^{-\rho\left(r_{i}\right)}\right)^{N}+\left(I-e^{-\rho\left(r_{i}\right) L}\right)^{N}\right) b_{i}\right) \\
& \leq S_{h}^{\rho}\left(\sum_{i}\left(I-\left(I-e^{-\rho\left(r_{i}\right)}\right)^{N}\right) b_{i}\right)+S_{h}^{\rho}\left(\sum_{i}\left(I-e^{-\rho\left(r_{i}\right)}\right)^{N} b_{i}\right) .
\end{aligned}
$$

Here $N \in \mathbb{N}$ is chosen to be larger than $2 v / \beta_{1}$, where $v$ is as in (1.5).

Then it is enough to prove that

$$
\begin{aligned}
& \mu\left(\left\{x \in M: S_{h}^{\rho}(f)(x)>\lambda\right\}\right) \leq \mu\left(\left\{x \in M: S_{h}^{\rho}(g)(x)>\frac{\lambda}{3}\right\}\right) \\
& +\mu\left(\left\{x \in M: S_{h}^{\rho}\left(\sum_{i}\left(I-\left(I-e^{-\rho\left(r_{i}\right)}\right)^{N}\right) b_{i}\right)(x)>\frac{\lambda}{3}\right\}\right) \\
& +\mu\left(\left\{x \in M: S_{h}^{\rho}\left(\sum_{i}\left(I-e^{-\rho\left(r_{i}\right) L}\right)^{N} b_{i}\right)(x)>\frac{\lambda}{3}\right\}\right) \lesssim \frac{1}{\lambda^{p_{0}}} \int|f(x)|^{p_{0}} d \mu(x) .
\end{aligned}
$$

We treat $g$ in a routine way. Since $S_{h}^{\rho}$ is $L^{2}$ bounded as shown in (1.11), then

$$
\mu\left(\left\{x \in M: S_{h}^{\rho}(g)(x)>\frac{\lambda}{3}\right\}\right) \lesssim \lambda^{-2}\|g\|_{2}^{2} \lesssim \lambda^{-p_{0}}\|g\|_{p_{0}} \lesssim \lambda^{-p_{0}}\|f\|_{p_{0}} .
$$

Now for the second term. Note that $I-\left(I-e^{-\rho\left(r_{i}\right) L}\right)^{N}=\sum_{k=1}^{N}(-1)^{k+1}\left(\begin{array}{c}N \\ k\end{array}\right) e^{-k \rho\left(r_{i}\right) L}$, it is enough to show that for every $1 \leq k \leq N$,

$$
\mu\left(\left\{x \in M: S_{h}^{\rho}\left(\sum_{i} e^{-k \rho\left(r_{i}\right) L} b_{i}\right)(x)>\frac{\lambda}{3 N}\right\}\right) \lesssim \frac{1}{\lambda^{p_{0}}} \int|f(x)|^{p_{0}} d \mu(x) .
$$

Note the following slight improvement of (2.2): for every $1 \leq k \leq N$ and for every $j \geq 1$, we have

$$
\left\|e^{-k \rho\left(r_{i}\right) L} b_{i}\right\|_{L^{2}\left(C_{j}\left(B_{i}\right)\right)} \lesssim \frac{2^{j v}}{\mu^{\frac{1}{p_{0}}-\frac{1}{2}}\left(B_{i}\right)} e^{-c_{k} 2^{j \tau\left(k \rho\left(r_{i}\right)\right)}}\left\|b_{i}\right\|_{L^{p_{0}}\left(B_{i}\right)} .
$$


Here $\tau(r)=\beta_{1} /\left(\beta_{1}-1\right)$ if $0<r<1$, otherwise $\tau(r)=\beta_{2} /\left(\beta_{2}-1\right)$. Indeed, it is obvious for $r_{i} \geq 1$ and $0<r_{i}<k^{-\frac{1}{\beta_{1}}}$. For $k^{-\frac{1}{\beta_{1}}} \leq r_{i}<1$, that is, $k \rho\left(r_{i}\right) \geq 1$, then $\left(\frac{\left(2^{j} r_{i} \beta_{2}\right.}{k \rho\left(r_{i}\right)}\right)^{\frac{1}{\beta_{2}-1}} \simeq 2^{j \frac{\beta_{2}}{\beta_{2}-1}}=2^{j \tau\left(k \rho\left(r_{i}\right)\right)}$.

With the above preparations, we can show (4.2) now. Write

$$
\mu\left(\left\{x:\left|S_{h}^{\rho}\left(\sum_{i} e^{-k \rho\left(r_{i}\right) L} b_{i}\right)(x)\right|>\frac{\lambda}{3 N}\right\}\right) \lesssim \frac{1}{\lambda^{2}}\left\|\sum_{i} e^{-k \rho\left(r_{i}\right) L} b_{i}\right\|_{2}^{2}
$$

By a duality argument,

$$
\begin{aligned}
\left\|\sum_{i} e^{-k \rho\left(r_{i}\right) L} b_{i}\right\|_{2} & =\sup _{\|\phi\|_{2}=1} \int_{M}\left|\sum_{i} e^{-k \rho\left(r_{i}\right) L} b_{i}\right||\phi| d \mu \leq \sup _{\|\phi\|_{2}=1} \sum_{i} \sum_{j=1}^{\infty} \int_{C_{j}\left(B_{i}\right)}\left|e^{-k \rho\left(r_{i}\right) L} b_{i}\right||\phi| d \mu \\
& =: \sup _{\|\phi\|_{2}=1} \sum_{i} \sum_{j=1}^{\infty} A_{i j} .
\end{aligned}
$$

Applying Cauchy-Schwarz inequality, (4.3) and (1.5), we get

$$
\begin{aligned}
A_{i j} & \leq\left\|e^{-k \rho\left(r_{i}\right) L} b_{i}\right\|_{L^{2}\left(C_{j}\left(B_{i}\right)\right)}\|\phi\|_{L^{2}\left(C_{j}\left(B_{i}\right)\right)} \\
& \lesssim 2^{\frac{3 j v}{2}} e^{-c 2^{j \tau\left(k \rho\left(r_{i}\right)\right)}} \mu\left(B_{i}\right)\left(\frac{1}{\mu\left(B_{i}\right)} \int_{B_{i}}\left|b_{i}\right|^{p_{0}} d \mu\right)^{\frac{1}{p_{0}}} \inf _{y \in B_{i}}\left(\mathcal{M}\left(|\phi|^{2}\right)(y)\right)^{1 / 2} \\
& \lesssim e^{-c 2^{j \tau\left(k \rho\left(r_{i}\right)\right)}} \mu\left(B_{i}\right) \inf _{y \in B_{i}}\left(\mathcal{M}\left(|\phi|^{2}\right)(y)\right)^{1 / 2} .
\end{aligned}
$$

Here $\mathcal{M}$ denotes the Hardy-Littlewood maximal operator:

$$
\mathcal{M} f(x)=\sup _{B \ni x} \frac{1}{\mu(B)} \int_{B}|f(x)| d \mu(x),
$$

where $B$ ranges over all balls containing $x$.

Then

$$
\begin{aligned}
\left\|\sum_{i} e^{-k \rho\left(r_{i}\right) L} b_{i}\right\|_{2} & \lesssim \lambda \sup _{\|\phi\|_{2}=1} \sum_{i} \sum_{j=1}^{\infty} e^{-c 2^{j \tau\left(k \rho\left(r_{i}\right)\right)}} \mu\left(B_{i}\right) \inf _{y \in B_{i}}\left(M\left(|\phi|^{2}\right)(y)\right)^{1 / 2} \\
& \lesssim \lambda \sup _{\|\phi\|_{2}=1} \int \sum_{i} \mathbb{1}_{B_{i}}(y)\left(\mathcal{M}\left(|\phi|^{2}\right)(y)\right)^{1 / 2} d \mu(y) \\
& \lesssim \lambda \sup _{\|\phi\|_{2}=1} \int_{\cup_{i} B_{i}}\left(\mathcal{M}\left(|\phi|^{2}\right)(y)\right)^{1 / 2} d \mu(y) \\
& \lesssim \lambda \mu^{1 / 2}\left(\cup_{i} B_{i}\right) \lesssim \lambda^{1-p_{0} / 2}\left(\int|f|^{p_{0}} d \mu\right)^{1 / 2} .
\end{aligned}
$$

The third inequality is due to the finite overlap of the Calderón-Zygmund decomposition. In the last line, for the first inequality, we use Kolmogorov's inequality (see for example [24, page 91]).

Therefore, we obtain

$$
\mu\left(\left\{x:\left|S_{h}^{\rho}\left(\sum_{i} e^{-k \rho\left(r_{i}\right) L} b_{i}\right)(x)\right|>\frac{\lambda}{3 N}\right\}\right) \lesssim \frac{1}{\lambda p_{0}} \int|f|^{p_{0}} d \mu .
$$

For the third term, we have

$$
\mu\left(\left\{x \in M: S_{h}^{\rho}\left(\sum_{i}\left(I-e^{-\rho\left(r_{i}\right) L}\right)^{N} b_{i}\right)(x)>\frac{\lambda}{3}\right\}\right)
$$


HARDY SPACES ON METRIC MEASURE SPACES WITH GENERALIZED SUB-GAUSSIAN HEAT KERNEL ESTIMATES7

$$
\leq \mu\left(\cup_{j} 4 B_{j}\right)+\mu\left(\left\{x \in M \backslash \cup_{j} 4 B_{j}: S_{h}^{\rho}\left(\sum_{i}\left(I-e^{-\rho\left(r_{i}\right) L}\right)^{N} b_{i}\right)(x)>\frac{\lambda}{3}\right\}\right) .
$$

From the Calderón-Zygmund decomposition and doubling volume property, we get

$$
\mu\left(\cup_{j} 4 B_{j}\right) \leq \sum_{j} \mu\left(4 B_{j}\right) \lesssim \sum_{j} \mu\left(B_{j}\right) \lesssim \frac{1}{\lambda^{p_{0}}}\|f\|_{p_{0}} .
$$

It remains to show that

$$
\Lambda:=\mu\left(\left\{x \in M \backslash \cup_{j} 4 B_{j}: S_{h}^{\rho}\left(\sum_{i}\left(I-e^{-\rho\left(r_{i}\right) L}\right)^{N} b_{i}\right)(x)>\frac{\lambda}{3}\right\}\right) \lesssim \frac{1}{\lambda^{p_{0}}} \int|f(x)|^{p_{0}} d \mu(x) .
$$

As a consequence of the Chebichev inequality, $\Lambda$ is dominated by

$$
\begin{aligned}
& \frac{9}{\lambda^{2}} \int_{M \backslash \cup_{j} 4 B_{j}}\left(S_{h}^{\rho}\left(\sum_{i}\left(I-e^{-\rho\left(r_{i}\right) L}\right)^{N} b_{i}\right)(x)\right)^{2} d \mu(x) \\
\leq & \frac{9}{\lambda^{2}} \int_{M \backslash \cup_{j} 4 B_{j}} \iint_{\Gamma(x)}\left(\sum_{i} \rho(t) L e^{-\rho(t) L}\left(I-e^{-\rho\left(r_{i}\right) L}\right)^{N} b_{i}(y)\right)^{2} \frac{d \mu(y)}{V(x, t)} \frac{d t}{t} d \mu(x) \\
\leq & \frac{18}{\lambda^{2}} \int_{M \backslash \cup_{j} 4 B_{j}} \iint_{\Gamma(x)}\left(\sum_{i} \mathbb{1}_{2 B_{i}}(y) \rho(t) L e^{-\rho(t) L}\left(I-e^{-\rho\left(r_{i}\right) L}\right)^{N} b_{i}(y)\right)^{2} \frac{d \mu(y)}{V(x, t)} \frac{d t}{t} d \mu(x) \\
& +\frac{18}{\lambda^{2}} \int_{M \backslash \cup_{j} 4 B_{j}} \iint_{\Gamma(x)}\left(\sum_{i} \mathbb{1}_{M \backslash 2 B_{i}}(y) \rho(t) L e^{-\rho(t) L}\left(I-e^{-\rho\left(r_{i}\right) L}\right)^{N} b_{i}(y)\right)^{2} \frac{d \mu(y)}{V(x, t)} \frac{d t}{t} d \mu(x) \\
= & \frac{18}{\lambda^{2}}\left(\Lambda_{\text {loc }}+\Lambda_{g l o b}\right) .
\end{aligned}
$$

For the estimate of $\Lambda_{l o c}$. Due to the bounded overlap of $2 B_{i}$, we can put the sum of $i$ out of the square up to a multiplicative constant. That is,

$$
\begin{aligned}
\Lambda_{l o c} & \lesssim \sum_{i} \int_{M \backslash \cup_{j} 4 B_{j}} \int_{0}^{\infty} \int_{B(x, t)}\left(\mathbb{1}_{2 B_{i}}(y) \rho(t) L e^{-\rho(t) L}\left(I-e^{-\rho\left(r_{i}\right) L}\right)^{N} b_{i}(y)\right)^{2} \frac{d \mu(y)}{V(x, t)} \frac{d t}{t} d \mu(x) \\
& \lesssim \sum_{i} \int_{M \backslash \cup_{j} 4 B_{j}} \int_{2 r_{i}}^{\infty} \int_{B(x, t)}\left(\mathbb{1}_{2 B_{i}}(y) \rho(t) L e^{-\rho(t) L}\left(I-e^{-\rho\left(r_{i}\right) L}\right)^{N} b_{i}(y)\right)^{2} \frac{d \mu(y)}{V(x, t)} \frac{d t}{t} d \mu(x) \\
& \lesssim \sum_{i} \int_{2 r_{i}}^{\infty} \int_{M}\left(\int_{B(y, t)} \frac{d \mu(x)}{V(x, t)}\right)\left(\mathbb{1}_{2 B_{i}}(y) \rho(t) L e^{-\rho(t) L}\left(I-e^{-\rho\left(r_{i}\right) L}\right)^{N} b_{i}(y)\right)^{2} d \mu(y) \frac{d t}{t} \\
& \lesssim \sum_{i} \int_{2 r_{i}}^{\infty} \int_{2 B_{i}}\left(\rho(t) L e^{-\rho(t) L}\left(I-e^{-\rho\left(r_{i}\right) L}\right)^{N} b_{i}(y)\right)^{2} d \mu(y) \frac{d t}{t} .
\end{aligned}
$$

For the second inequality, note that for every $i, x \in M \backslash \cup_{j} 4 B_{j}$ means $x \notin 4 B_{i}$. Then $y \in 2 B_{i}$ and $d(x, y)<t$ imply that $t \geq 2 r_{i}$. Thus the integral is zero for every $i$ if $0<t<2 r_{i}$. We obtain the third inequality by using the Fubini theorem and (1.6).

Then by using (4.3), it follows

$$
\begin{aligned}
\Lambda_{l o c} & \lesssim \sum_{i} \int_{2 r_{i}}^{\infty} \int_{2 B_{i}}\left(\frac{\mu^{\frac{1}{p_{0}}-\frac{1}{2}}\left(B_{i}\right)}{V^{\frac{1}{p_{0}}-\frac{1}{2}}(y, t)} \frac{V^{\frac{1}{p_{0}}-\frac{1}{2}}(y, t)}{\mu^{\frac{1}{p_{0}}-\frac{1}{2}}\left(B_{i}\right)} \rho(t) L e^{-\rho(t) L}\left(I-e^{-\rho\left(r_{i}\right) L}\right)^{N} b_{i}(y)\right)^{2} d \mu(y) \frac{d t}{t} \\
& \lesssim \sum_{i} \int_{2 r_{i}}^{\infty} \int_{2 B_{i}}\left(\frac{V^{\frac{1}{p_{0}}-\frac{1}{2}}\left(y, 4 r_{i}\right)}{V^{\frac{1}{p_{0}}-\frac{1}{2}}(y, t)} \frac{V^{\frac{1}{p_{0}}-\frac{1}{2}}(y, t)}{\mu^{\frac{1}{p_{0}}-\frac{1}{2}}\left(B_{i}\right)} \rho(t) L e^{-\rho(t) L}\left(I-e^{-\rho\left(r_{i}\right) L}\right)^{N} b_{i}(y)\right)^{2} d \mu(y) \frac{d t}{t}
\end{aligned}
$$




$$
\begin{aligned}
& \lesssim \mu^{1-\frac{2}{p_{0}}}\left(B_{i}\right) \sum_{i} \int_{2 r_{i}}^{\infty}\left(\frac{4 r_{i}}{t}\right)^{\nu^{\prime}\left(\frac{2}{p_{0}}-1\right)}\left\|V^{\frac{1}{p_{0}}-\frac{1}{2}}(\cdot, t) \rho(t) L e^{-\rho(t) L}\left(I-e^{-\rho\left(r_{i}\right) L}\right)^{N} b_{i}\right\|_{2}^{2} \frac{d t}{t} \\
& \lesssim \mu^{1-\frac{2}{p_{0}}}\left(B_{i}\right) \sum_{i}\left\|\left(I-e^{-\rho\left(r_{i}\right) L}\right)^{N} b_{i}\right\|_{p_{0}}^{2} \\
& \lesssim \mu^{1-\frac{2}{p_{0}}}\left(B_{i}\right) \sum_{i}\left\|b_{i}\right\|_{p_{0}}^{2} \lesssim \lambda^{2} \sum_{i} \mu\left(B_{i}\right) \lesssim \lambda^{2-p_{0}} \int|f|^{p_{0}} d \mu .
\end{aligned}
$$

For the second inequality, we use the reverse doubling property (1.7). The third inequality follows from the $L^{p_{0}}-L^{2}$ boundedness of the operator $V^{\frac{1}{p_{0}}-\frac{1}{2}}(\cdot, t) \rho(t) L e^{-\rho(t) L}$ (see Lemma 2.3). Then by using the $L^{p_{0}}$ boundedness of the heat semigroup, we get the fourth inequality.

Now for the global part. We split the integral into annuli, that is,

$$
\begin{aligned}
\Lambda_{g l o b} & \leq \int_{M} \iint_{\Gamma(x)}\left(\sum_{i} \mathbb{1}_{M \backslash 2 B_{i}}(y) \rho(t) L e^{-\rho(t) L}\left(I-e^{-\rho\left(r_{i}\right) L}\right)^{N} b_{i}(y)\right)^{2} \frac{d \mu(y)}{V(x, t)} \frac{d t}{t} d \mu(x) \\
& \leq \int_{0}^{\infty} \int_{M} \int_{B(y, t)}\left(\sum_{i} \mathbb{1}_{M \backslash 2 B_{i}}(y) \rho(t) L e^{-\rho(t) L}\left(I-e^{-\rho\left(r_{i}\right) L}\right)^{N} b_{i}(y)\right)^{2} \frac{d \mu(x)}{V(x, t)} d \mu(y) \frac{d t}{t} \\
& \leq \int_{0}^{\infty} \int_{M}\left(\sum_{i} \mathbb{1}_{M \backslash 2 B_{i}}(y) \rho(t) L e^{-\rho(t) L}\left(I-e^{-\rho\left(r_{i}\right) L}\right)^{N} b_{i}(y)\right)^{2} d \mu(y) \frac{d t}{t} .
\end{aligned}
$$

In order to estimate the above $L^{2}$ norm, we use an argument of dualization. Take the supremum of all functions $h(y, t) \in L^{2}\left(M \times(0, \infty), \frac{d \mu d t}{t}\right)$ with norm 1 , then

$$
\begin{aligned}
& \Lambda_{\text {glob }}^{1 / 2} \leq\left(\int_{0}^{\infty} \int_{M}\left(\sum_{i} \mathbb{1}_{M \backslash 2 B_{i}}(y) \rho(t) L e^{-\rho(t) L}\left(I-e^{-\rho\left(r_{i}\right) L}\right)^{N} b_{i}(y)\right)^{2} d \mu(y) \frac{d t}{t}\right)^{1 / 2}
\end{aligned}
$$

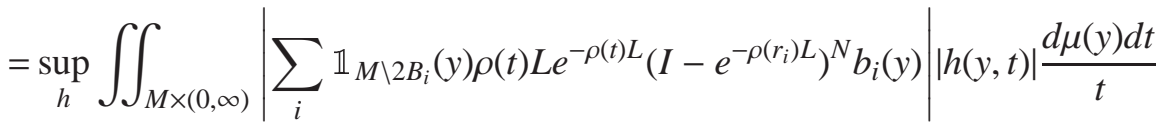

$$
\begin{aligned}
& \leq \sup _{h} \sum_{i} \sum_{j \geq 2} \int_{0}^{\infty} \int_{C_{j}\left(B_{i}\right)}\left|\rho(t) L e^{-\rho(t) L}\left(I-e^{-\rho\left(r_{i}\right) L}\right)^{N} b_{i}(y)\right||h(y, t)| \frac{d \mu(y) d t}{t} \\
& \leq \sup _{h} \sum_{i} \sum_{j \geq 2}\left(\int_{0}^{\infty} \int_{C_{j}\left(B_{i}\right)}\left|\rho(t) L e^{-\rho(t) L}\left(I-e^{-\rho\left(r_{i}\right) L}\right)^{N} b_{i}(y)\right|^{2} \frac{d \mu(y) d t}{t}\right)^{1 / 2} \\
& \times\left(\int_{0}^{\infty} \int_{C_{j}\left(B_{i}\right)}|h(y, t)|^{2} \frac{d \mu(y) d t}{t}\right)^{1 / 2} .
\end{aligned}
$$

Denote $I_{i j}=\left(\int_{0}^{\infty} \int_{C_{j}\left(B_{i}\right)}\left|\rho(t) L e^{-\rho(t) L}\left(I-e^{-\rho\left(r_{i}\right) L}\right)^{N} b_{i}(y)\right|^{2} \frac{d \mu(y) d t}{t}\right)^{1 / 2}$.

Let $H_{t, r}(\zeta)=\rho(t) \zeta e^{-\rho(t) \zeta}\left(1-e^{-\rho(r) \zeta}\right)^{N}$. Then

$$
I_{i j}=\left(\int_{0}^{\infty}\left\|H_{t, r_{i}}(L) b_{i}\right\|_{L^{2}\left(C_{j}\left(B_{i}\right)\right)}^{2} \frac{d t}{t}\right)^{1 / 2} .
$$

We will estimate $\left\|H_{t, r_{i}}(L) b_{i}\right\|_{L^{2}\left(C_{j}\left(B_{i}\right)\right)}$ by functional calculus. The notation is mainly taken from [2, Section 2.2]. 
For any fixed $t$ and $r$, then $H_{t, r}$ is a holomorphic function satisfying

$$
\left|H_{t, r}(\zeta)\right| \lesssim|\zeta|^{N+1}(1+|\zeta|)^{-2(N+1)},
$$

for all $\zeta \in \Sigma=\left\{z \in \mathbb{C}^{*}:|\arg z|<\xi\right\}$ with any $\xi \in(0, \pi / 2)$.

Since $L$ is a nonnegative self-adjoint operator, or equivalently $L$ is a bisectorial operator of type 0 , we can express $H_{t, r}(L)$ by functional calculus. Let $0<\theta<\omega<\xi<\pi / 2$, we have

$$
H_{t, r}(L)=\int_{\Gamma_{+}} e^{-z L} \eta_{+}(z) d z+\int_{\Gamma_{-}} e^{-z L} \eta_{-}(z) d z
$$

where $\Gamma_{ \pm}$is the half-ray $\mathbb{R}^{+} e^{ \pm i(\pi / 2-\theta)}$ and

$$
\eta_{ \pm}(z)=\int_{\gamma_{ \pm}} e^{\zeta z} H_{t, r}(\zeta) d \zeta, \forall z \in \Gamma_{ \pm}
$$

with $\gamma_{ \pm}$being the half-ray $\mathbb{R}^{ \pm} e^{ \pm i \omega}$.

Then for any $z \in \Gamma_{ \pm}$,

$$
\begin{aligned}
\left|\eta_{ \pm}(z)\right| & =\left|\int_{\gamma_{ \pm}} e^{\zeta z} \rho(t) \zeta e^{-\rho(t) \zeta}\left(1-e^{-\rho(r) \zeta}\right)^{N} d \zeta\right| \\
& \leq \int_{\gamma_{ \pm}}\left|e^{\zeta z-\rho(t) \zeta}\right| \rho(t)|\zeta|\left|1-e^{-\rho(r) \zeta}\right|^{N}|d \zeta| \\
& \leq \int_{\gamma_{ \pm}} e^{-c|\zeta|(|z|+\rho(t))} \rho(t)\left|\zeta \| 1-e^{-\rho(r) \zeta}\right|^{N}|d \zeta| \\
& \lesssim \int_{0}^{\infty} e^{-c s(|z|+\rho(t))} \rho(t) \rho^{N}(r) s^{N+1} d s \leq \frac{C \rho(t) \rho^{N}(r)}{(|z|+\rho(t))^{N+2}}
\end{aligned}
$$

In the second inequality, the constant $c>0$ depends on $\theta$ and $\omega$. Indeed, $\mathfrak{R}(\zeta z)=|\zeta||z| \mathfrak{R} e^{ \pm i(\pi / 2-\theta+\omega)}$. Since $\theta<\omega$, then $\pi / 2<\pi / 2-\theta+\omega<\pi$ and $\left|e^{\zeta z}\right|=e^{-c_{1}|\zeta||z|}$ with $c_{1}=-\cos (\pi / 2-\theta+\omega)$. Also it is obvious to see that $\left|e^{\rho(t) \zeta}\right|=e^{-c_{2} \rho(t)|\zeta|}$. Thus the second inequality follows. In the third inequality, let $\zeta=s e^{ \pm i \omega}$, we have $|d \zeta|=d s$. In addition, we dominate $\mid 1-e^{-\left.\rho(r) \zeta\right|^{N}}$ by $(\rho(r) \zeta)^{N}$.

We choose $\theta$ appropriately such that $|z| \sim \mathfrak{R}_{z}$ for $z \in \Gamma_{ \pm}$, then for any $j \geq 2$ fixed,

$$
\begin{aligned}
\left\|H_{t, r_{i}}(L) b_{i}\right\|_{L^{2}\left(C_{j}\left(B_{i}\right)\right)} & \lesssim\left(\int_{\Gamma_{+}}+\int_{\Gamma_{-}}\right)\left\|e^{-\mathfrak{R} z L} b_{i}\right\|_{L^{2}\left(C_{j}\left(B_{i}\right)\right)} \frac{\rho(t)}{(|z|+\rho(t))^{2}} \frac{\rho^{N}\left(r_{i}\right)}{(|z|+\rho(t))^{N}}|d z| \\
& \lesssim \int_{0}^{\infty}\left\|e^{-s L} b_{i}\right\|_{L^{2}\left(C_{j}\left(B_{i}\right)\right)} \frac{\rho(t) \rho^{N}\left(r_{i}\right)}{(s+\rho(t))^{N+2}} d s .
\end{aligned}
$$

Applying Lemma 2.3, then

$$
\begin{aligned}
\left\|H_{t, r_{i}}(L) b_{i}\right\|_{L^{2}\left(C_{j}\left(B_{i}\right)\right)} & \lesssim \frac{2^{j v}\left\|b_{i}\right\|_{p_{0}}}{\mu^{\frac{1}{2}-\frac{1}{p_{0}}}\left(B_{i}\right)} \int_{0}^{\infty} e^{-c\left(\frac{2^{j} r_{i}}{\rho^{-1}(s)}\right)^{\tau(s)}} \frac{\rho(t) \rho^{N}\left(r_{i}\right)}{(s+\rho(t))^{N+2}} d s \\
& \lesssim \frac{2^{j v}\left\|b_{i}\right\|_{p_{0}}}{\mu^{\frac{1}{2}-\frac{1}{p_{0}}}\left(B_{i}\right)}\left(\int_{0}^{\rho(t)}+\int_{\rho(t)}^{\infty}\right) e^{-c\left(\frac{2^{j} r_{i}}{\sigma(s)}\right)^{\tau(s)}} \frac{\rho(t) \rho^{N}\left(r_{i}\right)}{(s+\rho(t))^{N+2}} d s \\
& =: \frac{2^{j v}\left\|b_{i}\right\|_{p_{0}}}{\mu^{\frac{1}{2}-\frac{1}{p_{0}}}\left(B_{i}\right)}\left(H_{1}\left(t, r_{i}, j\right)+H_{2}\left(t, r_{i}, j\right)\right) .
\end{aligned}
$$

In the second and the third lines, $\tau(s)$ is originally defined in (4.3). In fact, it should be $\tau\left(\rho^{-1}(s)\right.$ ). Since $\rho^{-1}(s)$ and $s$ are unanimously larger or smaller than one, we always have $\tau(s)=\tau\left(\rho^{-1}(s)\right)$. 
Hence, by Minkowski inequality, we get from (4.5) and (4.6) that

$$
I_{i j} \lesssim \frac{2^{j v}\left\|b_{i}\right\|_{p_{0}}}{\mu^{\frac{1}{2}-\frac{1}{p_{0}}}\left(B_{i}\right)}\left(\left(\int_{0}^{\infty} H_{1}^{2}\left(t, r_{i}, j\right) \frac{d t}{t}\right)^{1 / 2}+\left(\int_{0}^{\infty} H_{2}^{2}\left(t, r_{i}, j\right) \frac{d t}{t}\right)^{1 / 2}\right) .
$$

It remains to estimate the two integrals $\int_{0}^{\infty} H_{1}^{2}\left(t, r_{i}, j\right) \frac{d t}{t}$ and $\int_{0}^{\infty} H_{2}^{2}\left(t, r_{i}, j\right) \frac{d t}{t}$. We claim that

$$
\int_{0}^{\infty} H_{1}^{2}\left(t, r_{i}, j\right) \frac{d t}{t}, \int_{0}^{\infty} H_{2}^{2}\left(t, r_{i}, j\right) \frac{d t}{t} \lesssim 2^{-2 \beta_{1} N j} .
$$

Estimate first $\int_{0}^{\infty} H_{1}^{2}\left(t, r_{i}, j\right) \frac{d t}{t}$. Since $\frac{\rho(t) \rho^{N}\left(r_{i}\right)}{(s+\rho(t))^{N+2}} \leq \frac{\rho^{N}\left(r_{i}\right)}{\rho(t)^{N+1}}$, we obtain

$$
H_{1}\left(t, r_{i}, j\right) \leq \int_{0}^{\rho(t)} e^{-c\left(\frac{2^{j} r_{i}}{\sigma(s)}\right)^{\beta_{2} /\left(\beta_{2}-1\right)}} \frac{\rho^{N}\left(r_{i}\right)}{\rho^{N+1}(t)} d s \lesssim e^{-c\left(\frac{2^{j} r_{i}}{t}\right)^{\beta_{2} /\left(\beta_{2}-1\right)}} \frac{\rho^{N}\left(r_{i}\right)}{\rho^{N}(t)} .
$$

It follows that

$$
\begin{aligned}
\int_{0}^{\infty} H_{1}^{2}\left(t, r_{i}, j\right) \frac{d t}{t} & \lesssim \int_{0}^{\infty} e^{-2 c\left(\frac{2^{j} r_{i}}{t}\right)^{\beta_{2} /\left(\beta_{2}-1\right)}} \frac{\rho^{2 N}\left(r_{i}\right)}{\rho^{2 N}(t)} \frac{d t}{t} \\
& \lesssim \int_{0}^{2^{j} r_{i}}\left(\frac{t}{2^{j} r_{i}}\right)^{c} \frac{\rho^{2 N}\left(r_{i}\right)}{\rho^{2 N}(t)} \frac{d t}{t}+\int_{2^{j} r_{i}}^{\infty} \frac{\rho^{2 N}\left(r_{i}\right)}{\rho^{2 N}(t)} \frac{d t}{t} \\
& \lesssim \frac{\rho^{2 N}\left(r_{i}\right)}{\rho^{2 N}\left(2^{j} r_{i}\right)} \lesssim 2^{-2 \beta_{1} N j}
\end{aligned}
$$

In the first inequality, we dominate the exponential term by polynomial one for the first integral, where $c$ in the second line is chosen to be larger than $2 \beta_{2} N$.

Now estimate $\int_{0}^{\infty} H_{2}^{2}\left(t, r_{i}, j\right) \frac{d t}{t}$. Write $\frac{\rho(t) \rho^{N}\left(r_{i}\right)}{(s+\rho(t))^{N+2}} \leq \frac{\rho(t) \rho^{N}\left(r_{i}\right)}{s^{N+2}}$. On the one hand,

$$
H_{2}\left(t, r_{i}, j\right)=\int_{\rho(t)}^{\infty} e^{-c\left(\frac{2^{j} r_{i}}{\sigma(s)}\right)^{\tau(s)}} \frac{\rho(t) \rho^{N}\left(r_{i}\right)}{(s+\rho(t))^{N+2}} d s \leq \int_{\rho(t)}^{\infty} \frac{\rho(t) \rho^{N}\left(r_{i}\right)}{s^{N+2}} d s=C \frac{\rho^{N}\left(r_{i}\right)}{\rho^{N}(t)} .
$$

On the other hand, we also have

$$
H_{2}\left(t, r_{i}, j\right) \lesssim 2^{-\beta_{1} N j} \frac{\rho(t)}{\rho\left(2^{j} r_{i}\right)}
$$

In fact,

$$
\begin{aligned}
H_{2}\left(t, r_{i}, j\right) & \leq \int_{\rho(t)}^{\infty} e^{-c\left(\frac{2^{j} r_{i}}{\sigma(s)}\right)^{\beta_{2} /\left(\beta_{2}-1\right)}} \frac{\rho(t) \rho^{N}\left(r_{i}\right)}{s^{N+1}} \frac{d s}{s} \\
& \lesssim 2^{-\beta_{1} N j} \frac{\rho(t)}{\rho\left(2^{j} r_{i}\right)} \int_{\rho(t)}^{\infty} e^{-c\left(\frac{2^{j} r_{i}}{\sigma(s)}\right)^{\beta_{2} /\left(\beta_{2}-1\right)}} \frac{\rho^{N+1}\left(2^{j} r_{i}\right)}{s^{N+1}} \frac{d s}{s} \\
& \lesssim 2^{-\beta_{1} N j} \frac{\rho(t)}{\rho\left(2^{j} r_{i}\right)} .
\end{aligned}
$$

Now we split the integral into two parts in the same way and control them by using (4.9) and (4.10) seperately. Then

$$
\begin{aligned}
\int_{0}^{\infty} H_{2}^{2}\left(t, r_{i}, j\right) \frac{d t}{t} & \lesssim \int_{0}^{2^{j} r_{i}} 2^{-2 \beta_{1} N j} \frac{\rho^{2}(t)}{\rho^{2}\left(2^{j} r_{i}\right)} \frac{d t}{t}+\int_{2^{j} r_{i}}^{\infty} \frac{\rho^{2 N}\left(r_{i}\right)}{\rho^{2 N}(t)} \frac{d t}{t} \\
& \lesssim 2^{-2 \beta_{1} N j} .
\end{aligned}
$$


Therefore, it follows from (4.7) and (4.8) that

$$
I_{i j} \lesssim \frac{\mu^{1 / 2}\left(2^{j} B_{i}\right)\left\|b_{i}\right\|_{p_{0}}}{\mu^{1 / p_{0}}\left(B_{i}\right)} 2^{-\beta_{1} N j} .
$$

Now for the integral $\left(\int_{0}^{\infty} \int_{C_{j}\left(B_{i}\right)}|h(y, t)|^{2} \frac{d \mu(y) d t}{t}\right)^{1 / 2}$. Take $\tilde{h}(y)=\int_{0}^{\infty}|h(y, t)|^{2} \frac{d t}{t}$, then

$$
\left(\int_{0}^{\infty} \int_{C_{j}\left(B_{i}\right)}|h(y, t)|^{2} \frac{d \mu(y) d t}{t}\right)^{1 / 2} \leq \mu^{1 / 2}\left(2^{j+1} B_{i}\right) \inf _{z \in B_{i}} \mathcal{M}^{1 / 2} \tilde{h}(z),
$$

where $\mathcal{M}$ is the Hardy-Littlewood maximal function.

Following the route for the proof of (4.2), we get from (4.11) and (4.12) that

$$
\begin{aligned}
\Lambda_{\text {glob }}^{1 / 2} & \lesssim \sup _{h} \sum_{i} \sum_{j \geq 2} \frac{2^{j v}\left\|b_{i}\right\|_{p_{0}}}{\mu^{\frac{1}{2}-\frac{1}{p_{0}}}\left(B_{i}\right)} 2^{-\beta_{1} N j} \mu^{1 / 2}\left(2^{j+1} B_{i}\right) \inf _{z \in B_{i}} \mathcal{M}^{1 / 2} \tilde{h}(z) \\
& \lesssim \lambda \sup _{h} \int_{M} \sum_{i} \mathbb{1}_{B_{i}}(y) \mathcal{M}^{1 / 2} \tilde{h}(y) d \mu(y) \\
& \lesssim \lambda \sup _{h} \int_{\cup_{i} B_{i}} \mathcal{M}^{1 / 2} \tilde{h}(y) d \mu(y) \\
& \lesssim \lambda \mu\left(\cup_{i} B_{i}\right)^{1 / 2} \lesssim \lambda^{1-p_{0} / 2} \int|f|^{p_{0}} d \mu .
\end{aligned}
$$

Here the supremum is taken over all the functions $h$ with $\|h\|_{L^{2}\left(\frac{d \mu d t}{t}\right)}=1$. Since $N>2 v / \beta_{1}$, the sum $\sum_{j \geq 2} 2^{-\beta_{1} N j+3 v j / 2}$ converges and we get the second inequality. The fourth one is a result of Kolmogorov's inequality.

Thus we have shown $\Lambda_{g l o b} \lesssim \lambda^{2-p_{0}} \int|f|^{p_{0}} d \mu$.

4.2. Counterexamples to $H_{L, S_{h}}^{p}(M)=L^{p}(M)$. Before moving forward to the proof of Theorem 1.8 , let us recall the following two theorems about the Sobolev inequality and the Green operator.

Theorem 4.2 ([18]). Let $(M, \mu)$ be a $\sigma$-finite measure space. Let $T_{t}$ be a semigroup on $L^{s}, 1 \leq$ $s \leq \infty$, with infinitesimal generator $-L$. Assume that $T_{t}$ is equicontinuous on $L^{1}$ and $L^{\infty}$. Then the following two conditions are equivalent:

(1) There exists $C>0$ such that $\left\|T_{t}\right\|_{1 \rightarrow \infty} \leq C t^{-D / 2}, \forall t \geq 1$.

(2) $T_{1}$ is from $L^{1}$ to $L^{\infty}$ and for $q>1, \exists C$ such that

$$
\begin{aligned}
& \qquad\|f\|_{p} \leq C\left(\left\|L^{\alpha / 2} f\right\|_{q}+\left\|L^{\alpha / 2} f\right\|_{p}\right), f \in \mathcal{D}\left(L^{\alpha / 2}\right), \\
& \text { where } 0<\alpha q<D \text { and } \frac{1}{p}=\frac{1}{q}-\frac{\alpha}{D} \text {. }
\end{aligned}
$$

Theorem 4.3. Let $M$ be a complete non-compact manifold. Then there exists a Green's function $G(x, y)$ which is smooth on $(M \times M) \backslash D$ satisfying

$$
\Delta_{x} \int_{M} G(x, y) f(y) d \mu(y)=f(x), \quad \forall f \in C_{0}^{\infty}(M) .
$$

For a proof, see for example [32].

We also observe that 
Lemma 4.4. Let $M$ be a Riemannian manifold satisfying the polynomial volume growth (1.13) and the two-sided sub-Gaussian heat kernel estimate $\left(H K_{2, m}\right)$. Let $B$ be an arbitrary ball with radius $r \geq 4$. Then there exists a constant $c>0$ depending on $d$ and $m$ such that for all $t$ with $r^{m} / 2 \leq t \leq r^{m}$,

$$
\int_{B} p_{t}(x, y) d \mu(y) \geq c, \forall x \in B
$$

Proof. Note that for any $x, y \in B$, we have $t \geq r^{m} / 2 \geq 2 r \geq d(x, y)$. Then $\left(H K_{2, m}\right)$ yields

$$
\begin{aligned}
\int_{B} p_{t}(x, y) d \mu(y) & \geq \int_{B} \frac{c}{t^{d / m}} \exp \left(-C\left(\frac{d^{m}(x, y)}{t}\right)^{1 /(m-1)}\right) d \mu(y) \\
& \geq \frac{c \mu(B)}{t^{d / m}} \exp \left(-C\left(\frac{r^{m}}{t}\right)^{1 /(m-1)}\right) \geq c
\end{aligned}
$$

Proof of Theorem 1.8. Let $\phi_{n} \in C_{0}^{\infty}(M)$ be a cut-off function as follows: $0 \leq \phi_{n} \leq 1$ and for some $x_{0} \in M$,

$$
\phi_{n}(x)=\left\{\begin{array}{lr}
1, & x \in B\left(x_{0}, n\right), \\
0, & x \in M \backslash B\left(x_{0}, 2 n\right) .
\end{array}\right.
$$

For simplicity, we denote $B\left(x_{0}, n\right)$ by $B_{n}$.

Taking $f_{n}=G \phi_{n}$, Theorem 4.3 says that $\Delta f_{n}=\phi_{n}$.

On the one hand, we apply Theorem 4.2 by choosing $T_{t}=e^{-t \Delta}$. Indeed, $e^{-t \Delta}$ is Markov hence bounded on $L^{p}$, equicontinuous on $L^{1}, L^{\infty}$ and satisfies

$$
\left\|e^{-t \Delta}\right\|_{1 \rightarrow \infty}=\sup _{x, y \in M} p_{t}(x, y) \leq C t^{-D / 2},
$$

where $D=2 d / m>2$. Then taking $\alpha=2$ and $p>\frac{D}{D-2}$, it follows that

$$
\left\|f_{n}\right\|_{p} \leq C\left(\left\|\Delta f_{n}\right\|_{q}+\left\|\Delta f_{n}\right\|_{p}\right),
$$

where $\frac{1}{p}=\frac{1}{q}-\frac{\alpha}{D}$, that is, $q=\frac{D p}{D+2 p}=\frac{d p}{d+m p}$.

Using the fact that $\Delta f_{n}=\phi_{n}$ and $\phi_{n} \leq \mathbb{1}_{B\left(x_{0}, 2 n\right)}$, we get

$$
\begin{aligned}
\left\|f_{n}\right\|_{p} & \lesssim\left(\left\|\phi_{n}\right\|_{\frac{d p}{d+m p}}+\left\|\phi_{n}\right\|_{p}\right) \lesssim\left(V^{\frac{d+m p}{d p}}\left(x_{0}, 2 n\right)+V^{\frac{1}{p}}\left(x_{0}, 2 n\right)\right) \\
& \lesssim\left(n^{m+d / p}+n^{d / p}\right) \lesssim n^{m+d / p} .
\end{aligned}
$$

In particular, $\left\|f_{n}\right\|_{2} \lesssim n^{m+d / 2}$.

On the other hand,

$$
\begin{aligned}
\left\|S_{h} f_{n}\right\|_{p}^{p} & =\int_{M}\left(\iint_{\Gamma(x)}\left|t^{2} \Delta e^{-t^{2} \Delta} f_{n}(y)\right|^{2} \frac{d \mu(y)}{V(x, t)} \frac{d t}{t}\right)^{p / 2} d \mu(x) \\
& =\int_{M}\left(\iint_{\Gamma(x)}\left|t^{2} e^{-t^{2} \Delta} \phi_{n}(y)\right|^{2} \frac{d \mu(y)}{V(x, t)} \frac{d t}{t}\right)^{p / 2} d \mu(x) .
\end{aligned}
$$

Since $\phi_{n} \geq \mathbb{1}_{B_{n}} \geq 0$, it follows from the Markovian property of the heat semigroup that

$$
\left\|S_{h} f_{n}\right\|_{p}^{p} \geq \int_{M}\left(\iint_{\Gamma(x)}\left|t^{2} e^{-t^{2} L_{1}} \mathbb{1}_{B_{n}}(y)\right|^{2} \frac{d \mu(y)}{V(x, t)} \frac{d t}{t}\right)^{p / 2} d \mu(x) .
$$


By using Lemma 4.4 , it holds that $e^{-t^{2} L_{\mathbb{1}}} B_{n / 2} \geq c$ if $\frac{n^{m / 2}}{2} \leq t \leq n^{m / 2}$. Then we get

$$
\left\|S_{h} f_{n}\right\|_{p}^{p} \gtrsim \int_{B\left(x_{0}, \frac{n^{m / 2}}{4}\right)}\left(\int_{\frac{m^{m} / 2}{2}}^{n^{m / 2}} \int_{B(x, t) \cap B_{n / 2}} \frac{t^{3}}{V(x, t)} d \mu(y) d t\right)^{p / 2} d \mu(x) .
$$

Observe also that, for $t>\frac{n^{m / 2}}{2}$ and $x \in B\left(x_{0}, \frac{n^{m / 2}}{4}\right)$, we have $B_{n} \subset B(x, t)$ as long as $n$ is large enough. Then the volume growth (1.13) gives us a lower bound in terms of $n$. That is,

$$
\left\|S_{h} f_{n}\right\|_{p}^{p} \gtrsim \int_{B\left(x_{0}, \frac{m^{m / 2}}{4}\right)}\left(\int_{\frac{n^{m / 2}}{2}}^{n^{m / 2}} \frac{\mu\left(B_{n}\right) t^{3}}{V\left(x, n^{m / 2}\right)} d t\right)^{p / 2} d \mu(x) \gtrsim n^{\frac{m d}{2}\left(1-\frac{p}{2}\right)} n^{m p+d p / 2} .
$$

Comparing the upper bound of $\left\|f_{n}\right\|_{p}$ in (4.15) for $p>\frac{D}{D-2}$, we obtain

$$
\left\|S_{h} f_{n}\right\|_{p} \gtrsim n^{\frac{m d}{2}\left(\frac{1}{p}-\frac{1}{2}\right)+m+\frac{d}{2}} \gtrsim n^{d\left(\frac{m}{2}-1\right)\left(\frac{1}{p}-\frac{1}{2}\right)}\left\|f_{n}\right\|_{p},
$$

where $p>\frac{D}{D-2}$.

Assume $D>4$, i.e. $m<d / 2$, we have $\frac{D}{D-2}<2$. Then for $\frac{D}{D-2}<p<2$, since $m>2$,

$$
n^{d\left(\frac{m}{2}-1\right)\left(\frac{1}{p}-\frac{1}{2}\right)} \rightarrow \infty \text { as } n \rightarrow \infty \text {. }
$$

Thus (4.16) implies that $L^{p} \subset H_{S_{h}}^{p}$ is not true for $p \in\left(\frac{D}{D-2}, 2\right)$, i.e. $p \in\left(\frac{d}{d-m}, 2\right)$, where $2<m<$ $d / 2$.

Our conclusion is: for any fixed $p \in\left(\frac{d}{d-m}, 2\right)$, according to (4.15) and (4.16), there exists a family of functions $\left\{g_{n}=\frac{f_{n}}{n^{m+d / p}}\right\}_{n \geq 1}$ such that $\left\|g_{n}\right\|_{p} \leq C,\left\|g_{n}\right\|_{2} \leq n^{\frac{d}{2}-\frac{d}{p}} \rightarrow 0$ and $\left\|S_{h} g_{n}\right\|_{p} \geq$ $n^{d\left(\frac{m}{2}-1\right)\left(\frac{1}{p}-\frac{1}{2}\right)} \rightarrow+\infty$ as $n$ goes to infinity. Therefore $S_{h}$ is not $L^{p}$ bounded for $p \in\left(\frac{d}{d-m}, 2\right)$ and the inclusion $L^{p} \subset H_{S_{h}^{m^{\prime}}}^{p}$ doesn't hold for $p \in\left(\frac{d}{d-m}, 2\right)$.

More generally, a slight adaption of Theorem 1.8 plus Theorem 1.6 yields the following result.

Corollary 4.5. Let $M$ be a Riemannian manifold satisfying (1.13) and $\left(H K_{2, m}\right)$ as above. Let $p \in$ $\left(\frac{d}{d-m}, 2\right)$. Then for any $0<m^{\prime} \leq m, L^{p}(M)=H_{S_{h}^{m^{\prime}}}^{p}(M)$ if and only if $m^{\prime}=m$.

Proof. If $m^{\prime}=m$, Theorem 1.6 says that $L^{p} \subset H_{S_{h}^{m}}^{p}$.

Conversely, by doing a slight adjustment for the above proof, we can show that $L^{p} \subset H_{S_{h}^{m^{\prime}}}^{p}$ is false for $p \in\left(\frac{d}{d-m}, 2\right)$, where $2<m<d / 2$ and $m^{\prime}<m$.

\section{The $H^{1}-L^{1}$ BOUNDEDNESS OF RIESZ TRANSFORMS ON FRACTAL MANIFOLDS}

This section is devoted to an application of the Hardy space theory we introduced above.

Let $(M, d, \mu)$ be a Riemannian manifold satisfying the doubling volume property $(D)$ and the sub-Gaussian estimate $\left(U E_{2, m}\right)$. Note that we could as well consider a metric measure Dirichlet space which admits a "carré du champ" (see, for example, [6, 26]).

Recall that the Riesz transform $\nabla \Delta^{-1 / 2}$ is of weak type $(1,1)$ on $M$ :

Theorem 5.1 ([14]). Let $M$ be a manifold satisfying the doubling volume property $(D)$ and the heat kernel estimate $\left(U E_{2, m}\right), m>2$. Then, the Riesz transform is weak $(1,1)$ bounded and bounded on $L^{p}$ for $1<p \leq 2$.

The proof depends on the following integrated estimate for the gradient of the heat kernel. 
Lemma 5.2 ([14]). Let $M$ be as above. Then for all $y \in M$, all $r, t>0$,

$$
\int_{M \backslash B(y, r)}\left|\nabla_{x} h_{t}(x, y)\right| d \mu(x) \lesssim \frac{1}{\sqrt{t}} \exp \left(-c\left(\frac{\rho(r)}{t}\right)^{\frac{1}{m-1}}\right),
$$

where $\rho$ is defined in (1.4).

Our aim here is to prove Theorem 1.9. More specifically, we will show that the Riesz transform is $H_{\Delta, m, m o l}^{1}(M)-L^{1}(M)$ bounded. Due to Theorem 1.5, it is $H_{\Delta, m}^{1}(M)-L^{1}(M)$ bounded. The method we use is similar as in [29, Theorem 3.2]. Note that the pointwise assumption $\left(U E_{\rho}\right)$ simplifies the proof below.

Note first the following lemma, which is crucial in our proof.

Lemma 5.3. Let $M$ be as above and let $p \in(1,2)$. Then for any $E, F \subset M$ and for any $n \in \mathbb{N}$, we have

$$
\left\|\nabla \Delta^{n} e^{-t \Delta} f \mid\right\|_{L^{p}(F)} \lesssim\left\{\begin{array}{lr}
\frac{1}{t^{n+1 / 2}} e^{-c \frac{d^{2}(E, F)}{t}\|f\|_{L^{p}(E)},} & 0<t<1, \\
\frac{1}{t^{n+1 / 2}} e^{-c\left(\frac{d^{m}(E, F)}{t}\right)^{1 /(m-1)}}\|f\|_{L^{p}(E)}, & t \geq 1 ;
\end{array}\right.
$$

where $f \in L^{p}(M)$ is supported in $E$. Consequently,

$$
\left\|\left|\nabla \Delta^{n} e^{-t \Delta} f\right|\right\|_{L^{p}(F)} \lesssim \frac{1}{t^{n+1 / 2}} e^{-c\left(\frac{\rho(d(E, F))}{t}\right)^{1 /(m-1)}}\|f\|_{L^{p}(E)} .
$$

Remark 5.4. To prove the lemma, it is enough to show that the following two estimates:

$$
\left\|\left|\nabla e^{-t \Delta} f\right|\right\|_{L^{p}(F)} \lesssim\left\{\begin{array}{lr}
e^{-c \frac{d^{2}(E, F)}{t}}\|f\|_{L^{p}(E)}, & 0<t<1, \\
e^{-c\left(\frac{d^{m}(E, F)}{t}\right)^{1 /(m-1)}}\|f\|_{L^{p}(E)}, & t \geq 1,
\end{array}\right.
$$

and

$$
\left\|(t \Delta)^{n} e^{-t \Delta} f\right\|_{L^{p}(F)} \lesssim\left\{\begin{array}{lr}
e^{-c \frac{d^{2}(E, F)}{t}\|f\|_{L^{p}(E)},} & 0<t<1, \\
e^{-c\left(\frac{d^{m}(E, F)}{t}\right)^{1 /(m-1)}}\|f\|_{L^{p}(E)}, & t \geq 1 .
\end{array}\right.
$$

Then (5.2) follows by adapting the proof of [30, Lemma 2.3]. Note that the first estimate can be obtained by using Stein's approach, similarly as the proof of Lemma 5.2. The second estimate is a direct consequence of $\left(U E_{\rho}\right)$ and the analyticity of the heat semigroup (see [23] for its discrete analogue). We omit the details of the proof here.

Remark 5.5. Note that (5.2) implies (5.3) (see [14, Corollary 2.4]), which may simplify the calculation in the subsequent proofs.

Proof of Theorem 1.9. Denote by $T:=\nabla \Delta^{-1 / 2}$. It suffices to show that, for any $(1,2, \varepsilon)$-molecule $a$ associated to a function $b$ and a ball $B$ with radius $r_{B}$, there exists a constant $C$ such that $\|T a\|_{L^{1}(M)} \leq$ C.

Write

$$
T a=T e^{-\rho\left(r_{B}\right) \Delta} a+T\left(I-e^{-\rho\left(r_{B}\right) \Delta}\right) a .
$$

Then

$$
\|T a\|_{L^{1}(M)} \leq\left\|T\left(I-e^{-\rho\left(r_{B}\right) \Delta}\right) a\right\|_{L^{1}(M)}+\left\|T e^{-\rho\left(r_{B}\right) \Delta} a\right\|_{L^{1}(M)}=: I+I I .
$$

We first estimate $I$. It holds that

$$
I \leq \sum_{i \geq 1}\left\|T\left(I-e^{-\rho\left(r_{B}\right) \Delta}\right) \mathbb{1}_{C_{i}(B)} a\right\|_{L^{1}(M)}
$$


HARDY SPACES ON METRIC MEASURE SPACES WITH GENERALIZED SUB-GAUSSIAN HEAT KERNEL ESTIMATE 55

$$
\leq \sum_{i \geq 1}\left(\left\|T\left(I-e^{-\rho\left(r_{B}\right) \Delta}\right) \mathbb{1}_{C_{i}(B)} a\right\|_{L^{1}\left(M \backslash 2^{i+2} B\right)}+\left\|T\left(I-e^{-\rho\left(r_{B}\right) \Delta}\right) \mathbb{1}_{C_{i}(B)} a\right\|_{L^{1}\left(2^{i+2} B\right)}\right)
$$

Using the Cauchy-Schwarz inequality and the $L^{2}$ boundedness of $T$ and $e^{-\rho\left(r_{B}\right) \Delta}$, it follows that

$$
\left\|T\left(I-e^{-\rho\left(r_{B}\right) \Delta}\right) \mathbb{1}_{C_{i}(B)} a\right\|_{L^{1}\left(2^{i+2} B\right)} \lesssim V\left(2^{i+2} B\right)\|a\|_{L^{2}\left(C_{i}(B)\right)} \lesssim 2^{-i \varepsilon} .
$$

Now we claim:

$$
\left\|T\left(I-e^{-\rho\left(r_{B}\right) \Delta}\right) \mathbb{1}_{C_{i}(B)} a\right\|_{L^{1}\left(M \backslash 2^{i+2} B\right)} \lesssim 2^{-i \varepsilon} .
$$

Combining (5.5) and (5.6), we obtain that $I$ is bounded.

In order to prove (5.6), we adapt the trick in [14]. For the sake of completeness, we write it down. First note that the spectral theorem gives us $\Delta^{-1 / 2} f=c \int_{0}^{\infty} e^{-s \Delta} f \frac{d s}{\sqrt{s}}$. Therefore,

$$
\begin{aligned}
\Delta^{-1 / 2}\left(I-e^{-t \Delta}\right) a & =c \int_{0}^{\infty}\left(e^{-s \Delta}-e^{-\left(s+\rho\left(r_{B}\right)\right) \Delta}\right) a \frac{d s}{\sqrt{s}} \\
& =c \int_{0}^{\infty}\left(\frac{1}{\sqrt{s}}-\frac{\chi\left\{s>\rho\left(r_{B}\right)\right\}}{\sqrt{s-\rho\left(r_{B}\right)}}\right) e^{-s \Delta} a d s .
\end{aligned}
$$

Set

$$
k_{\rho\left(r_{B}\right)}(x, y)=\int_{0}^{\infty}\left|\frac{1}{\sqrt{s}}-\frac{\chi\left\{s>\rho\left(r_{B}\right)\right\}}{\sqrt{s-\rho\left(r_{B}\right)}}\right|\left|\nabla_{x} h_{s}(x, y)\right| d s .
$$

Then

$$
\begin{aligned}
\left\|T\left(I-e^{-\rho\left(r_{B}\right) \Delta}\right) \mathbb{1}_{C_{i}(B)} a\right\|_{L^{1}\left(M \backslash 2^{i+2} B\right)} & \lesssim \int_{\left.M \backslash 2^{i+2} B\right)} \int_{C_{i}(B)} k_{\rho\left(r_{B}\right)}(x, y)|a(y)| d \mu(y) d \mu(x) \\
& \lesssim \int_{C_{i}(B)}|a(y)| \int_{d(x, y) \geq 2^{i} r} k_{\rho\left(r_{B}\right)}(x, y) d \mu(x) d \mu(y) .
\end{aligned}
$$

It remains to show that $\int_{d(x, y) \geq 2^{i} r} k_{\rho\left(r_{B}\right)}(x, y) d \mu(x)$ converges uniformly. Indeed, Lemma 5.2 yields

$$
\begin{aligned}
\int_{d(x, y) \geq 2^{i} r} k_{\rho\left(r_{B}\right)}(x, y) d \mu(x) & =\int_{0}^{\infty}\left|\frac{1}{\sqrt{s}}-\frac{\chi_{\left\{s>\rho\left(r_{B}\right)\right\}}}{\sqrt{s-\rho\left(r_{B}\right)}}\right| \int_{d(x, y) \geq 2^{i} r}\left|\nabla_{x} h_{s}(x, y)\right| d \mu(x) d s \\
& \lesssim \int_{0}^{\infty}\left|\frac{1}{\sqrt{s}}-\frac{\chi_{\left\{s>\rho\left(r_{B}\right)\right\}}}{\sqrt{s-\rho\left(r_{B}\right)}}\right| \frac{1}{\sqrt{s}} \exp \left(-c\left(\frac{\rho\left(2^{i} r\right)}{s}\right)^{\frac{1}{m-1}}\right) d s \\
& \lesssim 1 .
\end{aligned}
$$

Now turn to estimate $I I$. We have

$$
\begin{aligned}
I I & =\left\|c \int_{0}^{\infty} \nabla e^{-\left(s+\rho\left(r_{B}\right)\right) \Delta} a \frac{d s}{\sqrt{s}}\right\|_{L^{1}(M)} \\
& \lesssim \int_{0}^{\rho\left(r_{B}\right)}\left\|\left|\nabla e^{-\left(s+\rho\left(r_{B}\right)\right) \Delta} a\right|\right\|_{L^{1}(M)} \frac{d s}{\sqrt{s}}+\int_{\rho\left(r_{B}\right)}^{\infty}\left\|\nabla e^{-\left(s+\rho\left(r_{B}\right)\right) \Delta} \Delta^{K} b \mid\right\|_{L^{1}(M)} \frac{d s}{\sqrt{s}} \\
& =: I I_{1}+I I_{2} .
\end{aligned}
$$

We estimate $I I_{1}$ as follows:

$$
I I_{1} \leq \sum_{i \geq 1} \int_{0}^{\rho\left(r_{B}\right)}\left(\left\|\left|\nabla e^{-\left(s+\rho\left(r_{B}\right)\right) \Delta} \mathbb{1}_{C_{i}(B)} a\right|\right\|_{L^{1}\left(2^{i+2} B\right)}+\left\|\left|\nabla e^{-\left(s+\rho\left(r_{B}\right)\right) \Delta} \mathbb{1}_{C_{i}(B)} a\right|\right\|_{L^{1}\left(M \backslash 2^{i+2} B\right)}\right) \frac{d s}{\sqrt{s}} .
$$


Estimate the first term inside the sum by Cauchy-Schwarz and the fact that $\left\|e^{-t \Delta}\right\|_{2 \rightarrow 2} \lesssim \frac{1}{\sqrt{t}}$. Then

$$
\begin{aligned}
\int_{0}^{\rho\left(r_{B}\right)}\left\|\left|\nabla e^{-\left(s+\rho\left(r_{B}\right)\right) \Delta} \mathbb{1}_{C_{i}(B)} a\right|\right\|_{L^{1}\left(2^{i+2} B\right)} \frac{d s}{\sqrt{s}} & \lesssim \int_{0}^{\rho\left(r_{B}\right)} V^{1 / 2}\left(2^{i+2} B\right)\|a\|_{L^{2}\left(C_{i}(B)\right)} \frac{d s}{\sqrt{s+\rho\left(r_{B}\right)} \sqrt{s}} \\
& \lesssim 2^{-i \varepsilon} \int_{0}^{\rho\left(r_{B}\right)} \frac{d s}{\rho\left(r_{B}\right) \sqrt{s}} \\
& \lesssim 2^{-i \varepsilon}
\end{aligned}
$$

For the second term inside the sum, we use Lemma 5.2 again. Then

$$
\begin{aligned}
& \int_{0}^{\rho\left(r_{B}\right)}\left\|\left|\nabla e^{-\left(s+\rho\left(r_{B}\right)\right) \Delta} \mathbb{1}_{C_{i}(B)} a\right|\right\|_{L^{1}\left(M \backslash 2^{i+2} B\right)} \frac{d s}{\sqrt{s}} \\
& \lesssim \int_{0}^{\rho\left(r_{B}\right)} \int_{\left.M \backslash 2^{i+2} B\right)} \int_{C_{i}(B)}\left|\nabla p_{s+\rho\left(r_{B}\right)}(x, y) a(y)\right| d \mu(y) d \mu(x) \frac{d s}{\sqrt{s}} \\
& \lesssim \int_{0}^{\rho\left(r_{B}\right)} \int_{C_{i}(B)} \int_{d(x, y) \geq 2^{i+1} B}\left|\nabla p_{s+\rho\left(r_{B}\right)}(x, y)\right| d \mu(x)|a(y)| d \mu(y) \frac{d s}{\sqrt{s}} \\
& \lesssim\|a\|_{L^{1}\left(C_{i}(B)\right)} \int_{0}^{\rho\left(r_{B}\right)} \frac{d s}{\sqrt{s+\rho\left(r_{B}\right)} \sqrt{s}} \\
& \lesssim 2^{-i \varepsilon} .
\end{aligned}
$$

It remains to estimate $I I_{2}$. Using the same method as for $I I_{1}$, then

$$
I I_{2} \leq \sum_{i \geq 1} \int_{\rho\left(r_{B}\right)}^{\infty}\left(\left\|\left|\nabla e^{-\left(s+\rho\left(r_{B}\right)\right) \Delta} \Delta^{K} \mathbb{1}_{C_{i}(B)} b\right|\right\|_{L^{1}\left(2^{i+2} B\right)}+\left\|\left|\nabla e^{-\left(s+\rho\left(r_{B}\right)\right) \Delta} \Delta^{K} \mathbb{1}_{C_{i}(B)} b\right|\right\|_{L^{1}\left(M \backslash 2^{i+2} B\right)}\right) \frac{d s}{\sqrt{s}} .
$$

For the first term inside the sum, we estimate by using Cauchy-Schwartz inequality and the spectral theory. Then

$$
\begin{aligned}
\int_{\rho\left(r_{B}\right)}^{\infty}\left\|\left|\nabla \Delta^{K} e^{-\left(s+\rho\left(r_{B}\right)\right) \Delta_{1}} \mathbb{1}_{C_{i}(B)} b\right|\right\|_{L^{1}\left(2^{i+2} B\right)} \frac{d s}{\sqrt{s}} & \lesssim \int_{\rho\left(r_{B}\right)}^{\infty} \mu^{1 / 2}\left(2^{i+2} B\right)\left\|\left|\nabla \Delta^{K} e^{-\left(s+\rho\left(r_{B}\right)\right) \Delta_{1}} \mathbb{1}_{C_{i}(B)} b\right|\right\|_{L^{2}(M)} \frac{d s}{\sqrt{s}} \\
& \lesssim \int_{\rho\left(r_{B}\right)}^{\infty} \mu^{1 / 2}\left(2^{i+2} B\right)\|b\|_{L^{2}\left(C_{i}(B)\right)} \frac{d s}{\left(s+\rho\left(r_{B}\right)\right)^{K+1 / 2} \sqrt{s}} \\
& \lesssim 2^{-i \varepsilon} \rho^{K}\left(r_{B}\right) \int_{\rho\left(r_{B}\right)}^{\infty} \frac{d s}{s^{K+1}} \lesssim 2^{-i \varepsilon} .
\end{aligned}
$$

For the second term inside the sum, we use Lemma 5.3, then

$$
\begin{aligned}
& \int_{\rho\left(r_{B}\right)}^{\infty}\left\|\nabla \Delta^{K} e^{-\left(s+\rho\left(r_{B}\right)\right) \Delta} \mathbb{1}_{C_{i}(B)} b \mid\right\|_{L^{1}\left(M \backslash 2^{i+2} B\right)} \frac{d s}{\sqrt{s}} \\
& \lesssim \sum_{l=i+2}^{\infty} \int_{\rho\left(r_{B}\right)}^{\infty} \mu^{1 / p^{\prime}}\left(2^{l+1} B\right)\left\|\left|\nabla \Delta^{K} e^{-\left(s+\rho\left(r_{B}\right)\right) \Delta} \mathbb{1}_{C_{i}(B)} b\right|\right\|_{L^{p}\left(C_{l}(B)\right.} \frac{d s}{\sqrt{s}} \\
& \lesssim \sum_{l=i+2}^{\infty} \int_{\rho\left(r_{B}\right)}^{\infty} \mu^{1 / p^{\prime}}\left(2^{l+1} B\right) \exp \left(-c\left(\frac{\rho\left(d\left(C_{l}(B), C_{i}(B)\right)\right)}{s+\rho\left(r_{B}\right)}\right)^{1 /(m-1)}\right) \frac{\|b\|_{L^{p}\left(C_{i}(B)\right.} d s}{\sqrt{s}\left(s+\rho\left(r_{B}\right)\right)^{K+1 / 2}} \\
& \lesssim \sum_{l=i+2}^{\infty} 2^{-i \varepsilon} \rho^{K}\left(r_{B}\right)\left(\frac{\mu\left(2^{l+1} B\right)}{\mu\left(2^{i} B\right)}\right)^{1 / p^{\prime}} \int_{\rho\left(r_{B}\right)}^{\infty} \exp \left(-c\left(\frac{\rho\left(2^{l} r_{B}\right)}{s+\rho\left(r_{B}\right)}\right)^{1 /(m-1)}\right) \frac{d s}{\sqrt{s}\left(s+\rho\left(r_{B}\right)\right)^{K+1 / 2}} \\
& \lesssim \sum_{l=i+2}^{\infty} 2^{-i \varepsilon} \rho^{K}\left(r_{B}\right) 2^{(l-i) v / p^{\prime}} \int_{\rho\left(r_{B}\right)}^{\infty}\left(\frac{s}{\rho\left(2^{l} r_{B}\right)}\right)^{c} \frac{d s}{s^{K+1}}
\end{aligned}
$$




$$
\begin{aligned}
& \lesssim \sum_{l=i+2}^{\infty} 2^{-i \varepsilon} \rho^{K}\left(r_{B}\right) 2^{(l-i) v / p^{\prime}} \frac{1}{\rho^{c}\left(2^{l} r_{B}\right) \rho^{K-c}\left(r_{B}\right)} \\
& \lesssim 2^{-i \varepsilon} .
\end{aligned}
$$

This finishes the proof.

Acknowledgements: This work is part of the author's $\mathrm{PhD}$ thesis in cotutelle between the Laboratoire de Mathématiques, Université Paris-Sud and the Mathematical Science Institute, Australian National University. The author would like to thank Pascal Auscher for suggesting this topic, and to thank Thierry Coulhon for many discussions and suggestions. She is also grateful to Alan McIntosh and Dorothee Frey for helpful discussions. The author was partially supported by the ANR project "Harmonic analysis at its boundaries" ANR-12-BS01-0013 and the Australian Research Council (ARC) grant DP130101302.

\section{REFERENCES}

[1] Alex Amenta. Tent spaces over metric measure spaces under doubling and related assumptions. In Operator theory in harmonic and non-commutative analysis, volume 240 of Oper. Theory Adv. Appl., pages 1-29. Birkhäuser/Springer, Cham, 2014. 8

[2] P. Auscher. On necessary and sufficient conditions for $L^{p}$-estimates of Riesz transforms associated to elliptic operators on $\mathbb{R}^{n}$ and related estimates. Mem. Amer. Math. Soc., 186(871):xviii+75, 2007. 15, 18

[3] P. Auscher, S. Hofmann, and J.-M. Martell. Vertical versus conical square functions. Trans. Amer. Math. Soc., 364(10):5469-5489, 2012. 15

[4] P. Auscher, A. McIntosh, and A. Morris. Calderón reproducing formulas and applications to Hardy spaces. ArXiv e-prints, April 2013. arXiv:1304.0168. 2

[5] P. Auscher, A. McIntosh, and E. Russ. Hardy spaces of differential forms on Riemannian manifolds. J. Geom. Anal., 18(1):192-248, 2008. 2, 5, 10

[6] D. Bakry and Michel Émery. Diffusions hypercontractives. In Séminaire de probabilités, XIX, 1983/84, volume 1123 of Lecture Notes in Math., pages 177-206. Springer, Berlin, 1985. 23

[7] M. T. Barlow. Which values of the volume growth and escape time exponent are possible for a graph? Rev. Mat. Iberoamericana, 20(1):1-31, 2004. 6

[8] M. T. Barlow. Analysis on the Sierpinski carpet. In Analysis and geometry of metric measure spaces, volume 56 of CRM Proc. Lecture Notes, pages 27-53. Amer. Math. Soc., Providence, RI, 2013. 6

[9] M. T. Barlow and R. F. Bass. Stability of parabolic Harnack inequalities. Trans. Amer. Math. Soc., 356(4):15011533 (electronic), 2004. 7

[10] M. T. Barlow, T. Coulhon, and A. Grigor'yan. Manifolds and graphs with slow heat kernel decay. Invent. Math., 144(3):609-649, 2001. 6

[11] J. Bergh and J. Löfström. Interpolation spaces. An introduction. Springer-Verlag, Berlin, 1976. Grundlehren der Mathematischen Wissenschaften, No. 223. 8

[12] S. Blunck. Generalized Gaussian estimates and Riesz means of Schrödinger groups. J. Aust. Math. Soc., 82(2):149162, 2007. 15

[13] S. Blunck and P. C. Kunstmann. Generalized Gaussian estimates and the Legendre transform. J. Operator Theory, 53(2):351-365, 2005. 7

[14] L. Chen, T. Coulhon, J. Feneuil, and E. Russ. Riesz transform for $1 \leq p \leq 2$ without Gaussian heat kernel bound. ArXiv e-prints, October 2015. arXiv:1510.08275. 2, 3, 6, 23, 24, 25

[15] R. R. Coifman, Y. Meyer, and E. M. Stein. Some new function spaces and their applications to harmonic analysis. J. Funct. Anal., 62(2):304-335, 1985. 2, 8

[16] R. R. Coifman and G. Weiss. Analyse harmonique non-commutative sur certains espaces homogènes. Lecture Notes in Mathematics, Vol. 242. Springer-Verlag, Berlin, 1971. Étude de certaines intégrales singulières. 14

[17] R. R. Coifman and G. Weiss. Extensions of Hardy spaces and their use in analysis. Bull. Amer. Math. Soc., 83(4):569-645, 1977. 2

[18] T. Coulhon. Dimension à l'infini d'un semi-groupe analytique. Bull. Sci. Math., 114(4):485-500, 1990. 21

[19] T. Coulhon and A. Sikora. Gaussian heat kernel upper bounds via the Phragmén-Lindelöf theorem. Proc. Lond. Math. Soc. (3), 96(2):507-544, 2008. 7

[20] M. Cowling, I. Doust, A. McIntosh, and A. Yagi. Banach space operators with a bounded $H^{\infty}$ functional calculus. J. Austral. Math. Soc. Ser. A, 60(1):51-89, 1996. 15 
[21] X. T. Duong and A. McIntosh. Singular integral operators with non-smooth kernels on irregular domains. Rev. Mat. Iberoamericana, 15(2):233-265, 1999. 15

[22] C. Fefferman and E. M. Stein. $H^{p}$ spaces of several variables. Acta Math., 129(3-4):137-193, 1972. 2

[23] J. Feneuil. Riesz transform on graphs under subgaussian estimates. ArXiv e-prints, May 2015. arXiv:1505.07001. 3,24

[24] L. Grafakos. Classical Fourier analysis, volume 249 of Graduate Texts in Mathematics. Springer, New York, second edition, 2008. 16

[25] A. Grigor'yan. Heat kernel and analysis on manifolds, volume 47 of AMS/IP Studies in Advanced Mathematics. American Mathematical Society, Providence, RI, 2009. 4

[26] P. Gyrya and L. Saloff-Coste. Neumann and Dirichlet heat kernels in inner uniform domains. Astérisque, (336):viii+144, 2011. 23

[27] W. Hebisch and L. Saloff-Coste. On the relation between elliptic and parabolic Harnack inequalities. Ann. Inst. Fourier (Grenoble), 51(5):1437-1481, 2001. 6

[28] S. Hofmann, G. Lu, D. Mitrea, M. Mitrea, and L. Yan. Hardy spaces associated to non-negative self-adjoint operators satisfying Davies-Gaffney estimates. Mem. Amer. Math. Soc., 214(1007):vi+78, 2011. 2, 5, 8, 9, 12

[29] S. Hofmann and S. Mayboroda. Hardy and BMO spaces associated to divergence form elliptic operators. Math. Ann., 344(1):37-116, 2009. 24

[30] Steve Hofmann and José María Martell. $L^{p}$ bounds for Riesz transforms and square roots associated to second order elliptic operators. Publ. Mat., 47(2):497-515, 2003. 24

[31] P. C. Kunstmann and M. Uhl. Spectral multiplier theorems of Hörmander type on Hardy and Lebesgue spaces. $J$. Operator Theory, 73(1):27-69, 2015. 2, 5

[32] P. Li. Geometric analysis, volume 134 of Cambridge Studies in Advanced Mathematics. Cambridge University Press, Cambridge, 2012. 21

[33] E. Russ. The atomic decomposition for tent spaces on spaces of homogeneous type. In CMA/AMSI Research Symposium "Asymptotic Geometric Analysis, Harmonic Analysis, and Related Topics", volume 42 of Proc. Centre Math. Appl. Austral. Nat. Univ., pages 125-135. Austral. Nat. Univ., Canberra, 2007. 8

[34] E. M. Stein. Topics in harmonic analysis related to the Littlewood-Paley theory. Annals of Mathematics Studies, No. 63. Princeton University Press, Princeton, N.J., 1970. 15

[35] E. M. Stein. Harmonic analysis: real-variable methods, orthogonality, and oscillatory integrals, volume 43 of Princeton Mathematical Series. Princeton University Press, Princeton, NJ, 1993. With the assistance of Timothy S. Murphy, Monographs in Harmonic Analysis, III. 2

[36] K.-T. Sturm. Analysis on local Dirichlet spaces. I. Recurrence, conservativeness and $L^{p}$-Liouville properties. $J$. Reine Angew. Math., 456:173-196, 1994. 6

[37] K.-T. Sturm. Analysis on local Dirichlet spaces. II. Upper Gaussian estimates for the fundamental solutions of parabolic equations. Osaka J. Math., 32(2):275-312, 1995. 6

[38] M. Uhl. Spectral multiplier theorems of hörmander type via generalized gaussian estimates. Dissertation, Karlsruher Institut für Technologie (KIT), 2011. 2, 5, 7

[39] N. Th. Varopoulos. Long range estimates for Markov chains. Bull. Sci. Math. (2), 109(3):225-252, 1985.7

Li Chen, Mathematical Sciences Institute, The Australian National University, Canberra ACT 0200, Australia Current address: Instituto de Ciencias Matemáticas CSIC-UAM-UC3M-UCM, Consejo Superior de Investigaciones Científicas, C/ Nicolás Cabrera, 13-15, E-28049 Madrid, Spain

E-mail address: 1i.chen@icmat.es 